\title{
A HIGH-ORDER-ACCURATE GPU-BASED RADIATIVE TRANSFER EQUATION SOLVER FOR COMBUSTION AND PROPULSION APPLICATIONS
}

\author{
Xing $\mathrm{He}^{1}$, Euntaek Lee ${ }^{2}$, Lucas Wilcox ${ }^{1}$, \\ Ramakanth Munipalli ${ }^{1}$, and Laurent Pilon ${ }^{2}$ \\ ${ }^{1}$ HyPerComp, Inc., Westlake Village, California, USA \\ ${ }^{2}$ Mechanical and Aerospace Engineering Department, Henry Samueli School \\ of Engineering and Applied Science, University of California, Los Angeles, \\ California, USA
}

In this article we present a high-order-accurate solver for the radiative transfer equation (RTE) which uses the discontinuous Galerkin (DG) method and is designed for graphics processing units (GPUs). The compact nature of the high-order DG method enhances scalability, particularly on GPUs. High-order spatial accuracy can be used to reduce discretization errors on a given computational mesh, and can also reduce the mesh size needed to achieve a desired error tolerance. Computational efficiency is a key concern in solutions to radiative heat transfer problems, due to potentially large problem sizes created by (a) the presence of participating nongray media in a full-spectrum analysis, (b) the need to resolve a large number of angular directions and spatial extent of the domain for an accurate solution, and (c) potentially large variations in material and flow properties in the domain. We present here a simulation strategy, as well as a set of physical models, accompanied by a number of case studies, demonstrating the accuracy and superior performance in terms of computational efficiency of this approach.

\section{INTRODUCTION}

Thermal radiation is a dominant mode of heat transfer in combustion systems such as rocket engines, scramjets, and industrial furnaces [1-3]. It must be properly accounted for in preliminary and detailed design phases of systems development to result in a robust and fail-safe design. Hydrocarbon combustion results in exhaust gases which are dominantly composed of water vapor and carbon dioxide, both of which significantly participate in absorbing and emitting thermal radiation [4]. For

Received 9 November 2012; accepted 5 February 2013.

This work was supported by the United States Air Force under AFOSR STTR project grant \# F08A-T020-0170. The authors would like to thank Prof. Michael M. Modest from University of California, Merced, for his review of an earlier version of this manuscript. Thanks are also due to Dr. Zhining Liu of HyPerComp, Inc., who carried out the CFD simulations of the Atlas II rocket plume. The authors thank Prof. A. R. Karagozian for useful discussion and exchange of information.

Address correspondence to Laurent Pilon, Mechanical and Aerospace Engineering Department, Henry Samueli School of Engineering and Applied Science, University of California, Los Angeles, 420 Westwood Plaza, Los Angeles, CA 90095, USA. E-mail: pilon@seas.ucla.edu 


\begin{tabular}{|c|c|c|c|}
\hline \multicolumn{4}{|c|}{ NOMENCLATURE } \\
\hline$A$ & area, $\mathrm{m}^{2}$ & $\beta$ & extinction coefficient, $\mathrm{m}^{-1}$ \\
\hline$C$ & cross section, $\mathrm{m}^{2} /$ mole & $\epsilon$ & emmisivity \\
\hline$E$ & emissive power, $\mathrm{W} / \mathrm{m}^{2}$ & $\theta, \phi$ & spherical coordinate \\
\hline$F$ & blackbody distribution function, & $\kappa$ & absorption coefficient, $\mathrm{m}^{-1}$ \\
\hline$g$ & $\begin{array}{l}\text { Eq. (3) } \\
\text { asymmetric factor }\end{array}$ & $\sigma$ & $\begin{array}{l}\text { Stefan-Boltzmann constant, } \\
\mathrm{W} / \mathrm{m}^{2} \mathrm{~K}^{4}\end{array}$ \\
\hline$g_{i}$ & $\begin{array}{l}\text { cumulative distribution function of } \\
\text { absorption Eq. (8) }\end{array}$ & $\begin{array}{l}\sigma_{s} \\
\tau\end{array}$ & $\begin{array}{l}\text { scattering coefficient, } \mathrm{m}^{-1} \\
\text { optical thickness }\end{array}$ \\
\hline$G$ & fluence rate, $\mathrm{W} / \mathrm{m}^{2}$ & $\Phi$ & scattering phase function \\
\hline$H$ & height, $\mathrm{m}$ & $\omega$ & scattering albedo \\
\hline$I$ & radiation intensity, $\mathrm{W} / \mathrm{m}^{2} \mathrm{sr}$ & $\Omega$ & solid angle, sr \\
\hline$I_{b}$ & $\begin{array}{l}\text { blackbody radiation intensity, } \\
\mathrm{W} / \mathrm{m}^{2} \mathrm{sr}\end{array}$ & \multicolumn{2}{|c|}{ Croph } \\
\hline$k$ & absorption coefficient variable, $\mathrm{m}^{-1}$ & Subsc & \\
\hline$l$ & test function in DG method & abs & absorption \\
\hline$L$ & thickness, $m$ & $b$ & blackbody emission \\
\hline$N$ & number of grid elements & ext & external \\
\hline$N_{p}$ & $\begin{array}{l}\text { number of interpolation nodes inside } \\
\text { a grid element }\end{array}$ & $\begin{array}{l}g \\
\text { int }\end{array}$ & $\begin{array}{l}\text { gas properties } \\
\text { internal }\end{array}$ \\
\hline$P$ & pressure, $\mathrm{Pa}$ & $i, j$ & absorption coefficient indices \\
\hline$q$ & heat flux, $\mathrm{W} / \mathrm{m}^{2}$ & $n, m$ & grid element indices \\
\hline$r$ & position vector, $\mathrm{m}$ & $p$ & order of DG method \\
\hline$R$ & radius, $\mathrm{m}$ & $w$ & wall \\
\hline$s$ & path length, $m$ & $v$ & wavenumber \\
\hline$T$ & temperature, $\mathrm{K}$ & \\
\hline$V$ & volume, $\mathrm{m}^{3}$ & \multicolumn{2}{|c|}{ Superscripts } \\
\hline $\begin{array}{l}x, y, z \\
Y\end{array}$ & $\begin{array}{l}\text { cartesian coordinates, } \mathrm{m} \\
\text { molar fraction }\end{array}$ & $\begin{array}{l}+,- \\
*\end{array}$ & $\begin{array}{l}\text { adjacent and current grid elements } \\
\text { dimensionless }\end{array}$ \\
\hline
\end{tabular}

designing the rocket nozzle heat shield, quantities such as radiation heat load on the sidewalls, and, more importantly on the nozzle lip which directly faces the exhaust plume, are critical quantities which must be properly estimated [5]. Besides heating, accurate modeling of the thermal radiation emitted by the exhaust plume of a rocket or missile is of utmost importance for military applications for the design of low-observable vehicles and remote sensing [6].

Empirical correlations used in conjunction with experimental data have been used in the past for the design of such systems [4]. However, increasing complexity and cost has prompted the development of modeling and numerical simulation tools to better understand and design modern combustion systems. Numerical simulation of combustion systems is computationally complex since it involves multiphysics interactions, such as two-phase flow, turbulent mixing, fuel atomization and vaporization, radiative and convective heat transfer, and chemical reaction kinetics [1]. Furthermore, coupling radiative effects in such systems entails the computational burden of addressing multiscale physical phenomena. Flow, turbulence, combustion, scattering from particles, property gradients (with associated variations in optical thickness), and the propagation of radiation over long distances represent some multiscale aspects of the problem. Since the temperature field impacts combustion chemistry, resolution of temporal scales (of the order of microseconds or less) also adds to the computational 
complexity. Despite various advancements in numerical methods and computational hardware in recent times, the development of effective physical models and innovative numerical methods remains an important concern. This is particularly important when repeated coupled solutions of flow and radiation are desired in a time-evolving calculation, for e.g., in turbulent combustion and plasma dynamics.

General-purpose graphics processing units (GPGPUs) have been gaining increasing popularity in the high-performance computing community due to their enormous memory bandwidth and floating-point-operation capacity. It has also been demonstrated that computational solvers employing high-order schemes are able to exploit the full potential of GPGPUs [7].

This study presents a fast, robust, and accurate radiative transfer equation (RTE) solver implemented on a GPGPU based on a high-order discontinuous Galerkin method. It could find numerous applications in military and aerospace applications related to thermal analysis of combustion and propulsion systems and to target detection and identification (remote sensing) [2]. It could also be used in the analysis of exhaust gases in stationary power plants [8], radiative analysis of furnaces to minimize energy loss and pollution emissions [9], and even in simulation of light transfer in photobioreactors [10].

\section{CURRENT STATE OF KNOWLEDGE}

\subsection{Radiative Transfer Equation}

The radiative transfer equation describes the rate of change of the spectral radiation intensity along a line of sight in a participating medium. The gradient of intensity $I_{\nu}$ in a unit solid angle of $d \Omega$ (in sr) about the direction $\hat{\mathbf{s}}$ within a spectral range $d \nu$ about $\nu$ (in $\mathrm{cm}^{-1}$ ) can be expressed as [2]

$$
\begin{aligned}
\hat{\mathbf{s}} \cdot \nabla I_{\nu}(\mathbf{r}, \hat{\mathbf{s}})= & \kappa_{\nu}(\mathbf{r}) I_{b \nu}(\mathbf{r}, \hat{\mathbf{s}})-\kappa_{\nu}(\mathbf{r}) I_{\nu}(\mathbf{r}, \hat{\mathbf{s}})-\sigma_{s \nu}(\mathbf{r}) I_{\nu}(\mathbf{r}, \hat{\mathbf{s}}) \\
& +\frac{\sigma_{s \nu}(\mathbf{r})}{4 \pi} \int_{4 \pi} I_{\nu}(\mathbf{r}, \hat{\mathbf{s}}) \Phi_{\nu}\left(\hat{\mathbf{s}}_{i}, \hat{\mathbf{s}}\right) d \Omega_{i}
\end{aligned}
$$

where $\kappa_{v}\left(\right.$ in $\left.\mathrm{cm}^{-1}\right)$ is the spectral absorption coefficient, $\sigma_{s v}\left(\right.$ in $\left.\mathrm{cm}^{-1}\right)$ is the spectral scattering coefficient, $I_{\nu}$ is the radiation intensity expressed in $\mathrm{W} / \mathrm{m}^{2} \cdot \mathrm{sr} \cdot \mathrm{cm}, I_{b v}$ is the blackbody radiation intensity given by Planck's law. The scattering phase function $\Phi_{\nu}\left(\hat{\mathbf{s}}_{i}, \hat{\mathbf{s}}\right)$ represents the probability that radiation propagating in the solid angle $d \Omega_{i}$ direction around $\hat{\mathbf{s}}_{i}$ be scattered into the solid angle $d \boldsymbol{\Omega}$ around the direction $\hat{\mathbf{s}}$. In addition, $\beta_{\nu}\left(=\sigma_{s \nu}+\kappa_{\nu}\right)$ is the spectral extinction coefficient, while the single scattering albedo is defined as $\omega_{\nu}=\sigma_{s \nu} /\left(\sigma_{s \nu}+\kappa_{\nu}\right)$.

Spectrally and angularly integrated radiative quantities of interest in thermal science and in multiphysics problems include the total fluence rate $G(\mathbf{r})$, the total radiative heat flux $\mathbf{q}(\mathbf{r})$, and its divergence $\nabla \cdot \mathbf{q}(\mathbf{r})$, respectively defined as [3]

$$
\begin{aligned}
G(\mathbf{r}) & =\int_{4 \pi} \int_{0}^{\infty} I_{\nu}(\mathbf{r}, \hat{\mathbf{s}}) d \nu d \Omega, \quad \mathbf{q}(\mathbf{r})=\int_{4 \pi} \int_{0}^{\infty} \hat{\mathbf{s}} I_{\nu}(\mathbf{r}, \hat{\mathbf{s}}) d \nu d \Omega \\
\text { and } \quad \nabla \cdot \mathbf{q}(\mathbf{r}) & =\int_{4 \pi} \int_{0}^{\infty} \kappa_{\nu}(\mathbf{r})\left[I_{b \nu}(\mathbf{r})-I_{\nu}(\mathbf{r}, \hat{\mathbf{s}})\right] d \nu d \Omega
\end{aligned}
$$




\subsection{Gas Radiation Model}

The absorption coefficients of molecular gases vary significantly as function of temperature, pressure, and wavenumber [2]. This makes radiative transfer calculations in participating gases extremely difficult to carry out. Therefore, gas radiation models have been developed to determine the absorption coefficient $\kappa_{\nu}$ as a function of gas mixture composition, temperature, and pressure. Water vapor and carbon dioxide are of special importance in combustion of hydrocarbon fuels [2] and also dominate atmospheric radiation [11].

2.2.1. Spectral line-based weighted sum of gray gases (SLW). Denison and Webb [12-15] developed the spectral line-based weighted-sum-of-gray-gases (SLW) model. This model transforms spectral integration in Eq. (2) into a summation of a group of fictitious gray gases with specified absorption crosssection $C_{\mathrm{abs}}$ and weight $a_{j}$. For a medium with a participating gas of a specified uniform temperature $T_{g}$, molar fraction $Y_{i}$, and total pressure of gas mixture $P_{T}$, the mixture's absorption coefficient or the absorption cross-section can be determined as a function of wavenumber. The blackbody distribution function represents the fraction of blackbody emissive power at a particular temperature $T_{b}$ for wavenumber such that the cross-section $C_{\mathrm{abs}}^{\prime}\left(T_{g}, Y_{i}, P_{T}\right)$ is less than a specific value $C_{\mathrm{abs}}\left(T_{g}, Y_{i}\right.$, $P_{T}$ ). Mathematically, it can be represented as [14]

$$
F\left(T_{g}, T_{b}, C_{\mathrm{abs}}, Y_{i}, P_{T}\right)=\frac{1}{\sigma T_{b}^{4}} \int_{\nu, C_{\mathrm{abs}}^{\prime}<C_{\mathrm{abs}}} E_{b \nu}\left(\nu, T_{b}\right) d \nu
$$

where $\sigma\left(=5.67 \times 10^{-8} \mathrm{~W} / \mathrm{m}^{2} \mathrm{~K}^{4}\right)$ is the Stefan-Boltzmann constant. The weight of blackbody emissive power pertaining to the $j$ th fictitious gray-gas component is defined as [14]

$$
a_{j}=F\left(T_{g}, T_{b}, C_{\mathrm{abs}}, j+1, Y_{i}, P_{T}\right)-F\left(T_{g}, T_{b}, C_{\mathrm{abs}}, j, Y_{i}, P_{T}\right) \quad j=1, \ldots, N_{C_{\mathrm{abs}}}
$$

where $N_{C_{\text {abs }}}$ is the total number of the fictitious gray-gas components. Assuming the medium is nonscattering, the RTE along the $k$ th direction $\hat{s}_{k}$ becomes

$$
\hat{s}_{k} \cdot \nabla I_{j, k}=\kappa_{j}\left(I_{b, j}^{*}-I_{j, k}\right) \quad \text { with } j=1, \ldots, N_{C_{\mathrm{abs}}} \quad \text { and } \quad k=1, \ldots, N_{d}
$$

where $I_{b, j}^{*}=a_{j} I_{b, j}, N_{d}$ is total number of the angular directions, and $\kappa_{j}$ is the absorption coefficient of the $j$ th fictitious gray gas. Denison and Webb have constructed blackbody distribution functions from the HITRAN database [16] for water vapor [13] and carbon dioxide [14]. They also formulated the mathematical correlations between absorption line and distribution function for convenient computer code implementation. Treatments of nonisothermal and nonhomogeneous media were discussed in [15] by scaling approximation, with the help of reference temperature and the reference gas component fractions. Extension to multicomponent gas mixtures was realized by the multiplication approach described in [14, 17]. 


\subsection{Hybrid SNBCK Model}

The hybrid statistical narrow-band correlated- $k$ (SNBCK) model was proposed by Lacis and Oinas [18]. It was described in detail by Goutière et al. [19]. For the narrow-band gas model, the spectral domain is first divided into a series of narrow bands (of the order of $10-100 \mathrm{~cm}^{-1}$ ). The band width of the $i$ th narrow band is $\Delta \nu_{i}$, and the RTE for an absorbing, emitting, and nonscattering medium can be written as

$$
\hat{\mathbf{s}} \cdot \nabla I_{i}=\kappa_{i}\left(I_{b, i}-I_{i}\right)
$$

where $I_{i}=I_{i}(\hat{\mathbf{s}}, \mathbf{r})$ and $\kappa_{i}$ are the radiative intensity and absorption coefficient of the $i$ th narrow band, respectively. Note that $\kappa_{i}$ may still vary widely in this small spectral window, whereas the blackbody intensity $I_{b, i}$ is a smooth function in terms of spectral variable, which can be safely assumed to be constant inside $\Delta \nu_{i}$ around $\nu_{i}$. Integration of Eq. (6) over the spectral region $\Delta \nu_{i}$ yields

$$
\int_{\Delta \nu_{i}} \hat{\mathbf{s}} \cdot \nabla I_{i} d \nu=\int_{\Delta \nu_{i}} \kappa_{i}\left(I_{b, i}-I_{i}\right) d \nu
$$

A cumulative distribution function of absorption, $g_{i}(k)$, is introduced to represent the fraction in the spectral domain with absorption coefficient less than a specific value and is defined as

$$
g_{i}(k)=\int_{0}^{k} f_{i}\left(k^{\prime}\right) d k^{\prime}
$$

where $f_{i}\left(k^{\prime}\right)$ is the $k$-distribution function for the absorption coefficient in the spectral interval $\Delta v_{i}$. The function $g_{i}(k)$ is smooth and varies within $[0,1]$. Then, Eq. (7) can be written as

$$
\Delta \nu_{i} \int_{0}^{1} \hat{\mathbf{s}} \cdot \nabla I_{i} d g_{i}=\Delta \nu_{i} \int_{0}^{1} \kappa_{i}\left(I_{b, i}-I_{i}\right) d g_{i}
$$

Since the cumulative function is a smooth function, we can transform the integration into a quadrature summation, e.g., Gauss quadrature, with $N_{q}$ quadrature angles. Then, Eq. (9) becomes

$$
\Delta \nu_{i} \sum_{j=1}^{N_{q}}\left(w_{j} \hat{\mathbf{s}} \cdot \nabla I_{i, j}\right)=\Delta \nu_{i} \sum_{j=1}^{N_{q}} w_{j} \kappa_{i, j}\left(I_{b, i}-I_{i, j}\right)
$$

where $\kappa_{i, j}$ is the absorption coefficient at the $j$ th quadrature in the $i$ th narrow band and $w_{j}$ is the weight of the $j$ th Gauss quadrature.

Assuming that the quadrature directions are decoupled, then for each direction one can write [19]

$$
\hat{\mathbf{s}} \cdot \nabla I_{i, j}=\kappa_{i, j}\left(I_{b, i}-I_{i, j}\right) \quad j=1,2, \ldots, N_{q}
$$

In the SNBCK model, for example, we have 44 narrow bands $(i=1, \ldots, 44)$, with 7 Gauss quadrature points $(j=1, \ldots, 7)$ in each band. Databases of $\kappa_{i, j}$ for water vapor 
and carbon dioxide in the temperature range 300-2,500 $\mathrm{K}$ and pressure of 1 atm were tabulated by Soufiani and Taine [20].

\subsection{Discontinuous Galerkin Method for Solving the RTE}

The discontinuous Galerkin (DG) method was first used by Reed and Hill [21] to solve the neutron transport equation. It has been applied in recent years to a large number of problems in computational physics - see, e.g., Hesthaven and Warburton [22] for an exposition of the method. In the DG method the solution is expressed in terms of basis functions which are local to each element and can be discontinuous at element boundaries - as opposed to the traditional finite-element method, where interelement continuity of the basis is required. Lagrange polynomials are commonly used as the basis and test functions in the DG method [22]. A DG method of order $n$ is referred to as $P_{n}$. In our work, we use the $n$ th-order Lagrange polynomials as basis. High-order accuracy can be achieved by increasing $n$.

The DG method provides enormous numerical flexibility and is distinguished by the following features: (1) It is elementwise conservative [23]; (2) it can provide arbitrarily high-order accuracy and polynomials [23]; (3) adaptive variants of the scheme can be developed, where $n$ and mesh resolution may be refined locally [24, 25]; (4) the method can be applied to general mesh topologies [26]; and (5) it is well suited for parallel computing using graphics processing units (GPUs). This last property results from the observation that the high-order DG uses dense local operators, an aspect that is further explained in [7].

Cui and Li [23] employed the P-1 DG method for solving the RTE in an emitting, absorbing, and anisotropically scattering gray medium. The authors solved the 2-D and 3-D RTE in square, quadrilateral, and cubic geometries with unstructured triangle and tetrahedral elements. The walls were cold and black. They compared the wall heat flux obtained using the DG method with that obtained by ray-tracing and Monte Carlo methods and found excellent agreement. Moreover, Cui and Li [27] used the P-1 DG method for solving the 2-D RTE in an emitting, absorbing, and anisotropically scattering gray medium with axisymmetric geometries. The authors found good agreement between the heat flux obtained using the DG method and that predicted by the discrete-ordinates method and the finite-volume method.

Zhao and Liu [26] developed the discontinuous spectral-element method combining the different orders (P-1 to P-14) of the DG method with the spectral-element method [28] to solve the 2-D RTE. In the spectral-element method, orthogonal polynomials such as Chebyshev and Legendre polynomials were used as the interpolation functions of a numerical solution to achieve high-order accuracy [26]. The authors calculated the radiative heat flux for an emitting, absorbing, and anisotropically scattering gray medium within a 2-D square enclosure with black walls. They found that the maximum relative error in the radiative heat flux between the discontinuous spectral-element method and the discrete-ordinates method was less than $0.8 \%$.

Recently, Balima et al. [29] solved the 2-D RTE in a nonemitting, absorbing, isotropically scattering and gray medium using two finite-element formulations with the discrete-ordinates method. The authors compared the wall heat flux obtained by the least-square finite-element method and the P-1 DG method. They considered collimated incident radiation and evaluated the accuracy of two different angular 
quadrature sets, namely, $S_{N}$ and $T_{N}$. They concluded that the DG method with $T_{N}$ angular quadrature gave more accurate solutions than the least-square finite-element method with $S_{N}$ angular quadrature. Balima et al. [30] also employed the P-1 DG method for solving the 2-D RTE in the frequency domain for optical tomography. The medium was gray, nonemitting, absorbing, and anisotropically scattering. The authors also considered collimated incident radiation and used a gradient-based algorithm to retrieve the optical properties of the medium. They concluded that the DG method achieved good reconstruction of the optical properties distribution and was well suited for optical tomography.

\subsection{Graphics Processing Units}

The graphics processing unit is a highly parallel, multithreaded, and many-core processor (hundreds of cores) with enormous computing power. Its low cost, high floating-point-operation throughput, and memory access bandwidth have been attracting more and more researchers in the field of high-performance computing [7, 31-34]. In addition, compared with cluster systems that consist of many CPUs, GPU computing is low-cost and requires low energy at equivalent performance. Across many disciplines of science and engineering, users have been able to increase performance by several orders of magnitude using GPUs [35].

In the computational fluid dynamics (CFD) community, structured-grid Euler solvers for compressible flows on GPU were implemented by Elsen et al. [32]. Typically, one order of magnitude speed-up was achieved by using a single GPU card compared with a single-node CPU implementation. Philips et al. [34] implemented a parallel 2-D structured-grid Euler solver and used a GPU cluster consisting of 8 GPU cards, each having 128 cores. They achieved a speed-up of 160 compared with a single CPU implementation. It is worth mentioning that a recent study by Klöckner et al. [7] achieved a 40-times speed-up when solving Maxwell's equation solver with GPUs using the discontinuous Galerkin method and general unstructured 3-D grids.

In previous studies solving the RTE using the DG method, the medium was treated as gray and the walls as black. In this article, we significantly expand the scope of the prospective applications of the DG-based RTE solver by considering higher-order methods and by including the effects of nongray media and generalized boundary conditions, as well as by porting the method to GPU computing. In fact, high-order discontinuous Galerkin (DG) methods were used to solve the 3-D RTE on a spectral basis using the gas radiation models SLW and hybrid SNBCK. Diffuse and collimated incident radiation was considered. Black and diffusely or specularly reflecting walls were also used as boundary conditions to analyze various radiative transfer problems. Finally, the RTE solver was coupled with a CFD solver to simulate radiation transfer in Atlas II rocket plumes. The computational efficiencies of the parallel GPU and CPU were examined for integrating RTE and CFD solvers.

\section{ANALYSIS}

\subsection{Mathematical Details}

Efficiently solving the radiative transfer equation in a single direction, even without scattering term, is of critical importance for the overall performance of 
the RTE solver. For a given direction, the RTE in absorbing, emitting, but nonscattering media takes the following form:

$$
\hat{\mathbf{s}} \cdot \nabla I_{\nu}=-\kappa_{\nu} I_{\nu}+\kappa_{\nu} I_{b \nu}
$$

Under the DG framework [22], multiplying Eq. (12) with a test function $l_{m}^{n}$ and performing volume integration for the $n$th grid element of volume $V^{n}$ and surface area $A^{n}$ yields

$$
\int_{V^{n}} l_{m}^{n} \hat{\mathbf{s}} \cdot \nabla I^{n} d V+\int_{V^{n}} l_{m}^{n} \kappa I^{n} d V=\int_{V^{n}} l_{m}^{n} \kappa I_{b} d V
$$

where the superscript $n \in\{1, \ldots, N\}$ is used to indicate that the integration operation is carried out locally on the $n$th grid element, out of a total of $N$ grid elements, and $l_{m}^{n}$ is the $m$ th test function for the $n$th grid element $m \in\left\{1, \ldots, N_{p}\right\}$ where $N_{p}$ is the number of interpolation nodes. For the sake of simplicity, the spectral subscript $\nu$ was dropped, but it is understood that the radiative intensity and the radiation characteristics presented in all formulations of this sections are wavelength-dependent. The weak formulation is obtained by applying the Gauss (or divergence) theorem on the first term on the left-hand side (LHS) of Eq. (13) and is given by

$$
\int_{A^{n}} l_{m}^{n} \hat{\mathbf{s}} I^{*} \cdot d \boldsymbol{A}-\int_{V^{n}} \nabla l_{m}^{n} \cdot \hat{\mathbf{s}} I^{n} d V+\int_{V^{n}} l_{m}^{n} \kappa I^{n} d V=\int_{V^{n}} l_{m}^{n} \kappa I_{b} d V
$$

where $I^{*}$ is the numerical radiative intensity to be specified along the boundary of the element. Applying the Gauss theorem again on the second term on the LHS of Eq. (14) yields the strong formulation, expressed as

$$
\int_{V^{n}} l_{m}^{n} \hat{\mathbf{s}} \cdot \nabla I^{n} d V+\int_{V^{n}} l_{m}^{n} \kappa I^{n} d V+\int_{A^{n}} l_{m}^{n}\left(\hat{\mathbf{s}} I^{*}-\hat{\mathbf{s}} I^{-}\right) \cdot d \boldsymbol{A}=\int_{V^{n}} l_{m}^{n} \kappa I_{b} d V
$$

The derivation of the weak and strong formulations as well as the introduction of numerical flux through the element boundary is a standard procedure in the DG framework, as detailed in [22]. The numerical radiative heat flux through the grid element boundary $\hat{\mathbf{s}} I^{*}$ is a function of radiative intensity along both sides of the boundary. The upwind scheme was employed for numerical flux construction so that

$$
\hat{\mathbf{s}} I^{*}= \begin{cases}\hat{\mathbf{s}} I^{+} & \text {if } \hat{\mathbf{s}} \cdot \boldsymbol{A}<0 \\ \hat{\mathbf{s}} I^{-} & \text {if } \hat{\mathbf{s}} \cdot \boldsymbol{A}>0\end{cases}
$$

where $\boldsymbol{A}$ is the outward normal vector to the grid element surface area. The minus superscript $\left(^{-}\right)$refers to variables obtained from the grid element under consideration, while the positive superscript $\left(^{+}\right)$is used for variables from adjacent grid elements. Therefore, the third term on the LHS of Eq. (15) can be written as

$$
\int_{A^{n}} l_{m}^{n}\left(\hat{\mathbf{s}} I^{*}-\hat{\mathbf{s}} I^{-}\right) d \boldsymbol{A}=\frac{1}{2} \int_{A^{n}} l_{m}^{n}[1-\operatorname{sign}(\hat{\mathbf{s}} \cdot d \boldsymbol{A})]\left(I^{+}-I^{-}\right) \hat{\mathbf{s}} d \boldsymbol{A}
$$


where the sign function $\operatorname{sign}(x)$ is the sign of scalar $x$. Substituting Eq. (17) into Eq. (15) yields

$$
\begin{aligned}
& \int_{V^{n}} l_{m}^{n} \hat{\mathbf{s}} \cdot \nabla I^{n} d V+\int_{V^{n}} l_{m}^{n} \kappa I^{n} d V \\
& \quad+\frac{1}{2} \int_{A^{n}} l_{m}^{n}[1-\operatorname{sign}(\hat{\mathbf{s}} \cdot d \boldsymbol{A})]\left(I^{+}-I^{-}\right) \hat{\mathbf{s}} d \boldsymbol{A}=\int_{V^{n}} l_{m}^{n} \kappa I_{b} d V
\end{aligned}
$$

As previously noted, $m \in\left\{1, \ldots, N_{p}\right\}$ and there are $N_{p}$ relations for the $n$th grid element. Introducing the vector $L^{n}=\left[l_{1}^{n}, l_{2}^{n}, \ldots, l_{N_{p}}^{n}\right]^{T}$ and combining Eq. (15)-(18) yields

$$
\begin{aligned}
& \int_{V^{n}} L^{n} \hat{\mathbf{s}} \cdot \nabla I^{n} d V+\int_{V^{n}} L^{n} \kappa I^{n} d V \\
& \quad+\frac{1}{2} \int_{A^{n}} L^{n}[1-\operatorname{sign}(\hat{\mathbf{s}} \cdot d \boldsymbol{A})]\left(I^{+}-I^{-}\right) \hat{\mathbf{s}} d \boldsymbol{A}=\int_{V^{n}} L^{n} \kappa I_{b} d V
\end{aligned}
$$

Here, the mass matrix $M^{n}$ can be defined as the matrix of weights used to integrate polynomials whose elements $M_{m p}^{n}$ for the $n$th grid element are expressed as functions of Lagrange polynomials

$$
M_{m p}^{n}=\int_{V^{n}} l_{m}^{n} l_{p}^{n} d V
$$

The inverse of this mass matrix can be applied to Eq. (19) to yield

$$
\begin{aligned}
& \left(M^{n}\right)^{-1} \int_{V^{n}} L^{n} \hat{\mathbf{s}} \cdot \nabla I^{n} d V+\left(M^{n}\right)^{-1} \int_{V^{n}} L^{n} \kappa I^{n} d V \\
& \quad+\frac{1}{2}\left(M^{n}\right)^{-1} \int_{A^{n}} L^{n}[1-\operatorname{sign}(\hat{\mathbf{s}} \cdot d \boldsymbol{A})]\left(I^{+}-I^{-}\right) \hat{\mathbf{s}} d \boldsymbol{A}=\left(M^{n}\right)^{-1} \int_{V^{n}} L^{n} \kappa I_{b} d V
\end{aligned}
$$

The task of the RTE solver is to find $I^{n}=\sum_{m=1}^{N_{p}} l_{m}^{n} I_{m}^{n}$ satisfying Eq. (21), upon which $N \times N_{p}$ algebraic equations can be constructed. With these algebraic equations, in theory, the solution of the RTE of a particular direction can be found by inverting an $\left(N \times N_{p}\right) \times\left(N \times N_{p}\right)$ matrix. In our RTE solver, the matrix inversion was achieved using an iterative method [36].

The third term on the LHS of Eq. (21) is separated into internal and external boundary surfaces, since it might involve contribution from domain boundaries which need to be treated as a source term, i.e.,

$$
\int_{A^{n}}=\int_{A_{\mathrm{int}}^{n}}+\int_{A_{\mathrm{ext}}^{n}}
$$


We further separate the external boundary surface integration into

$$
\begin{aligned}
& \frac{1}{2}(\left.M^{n}\right)^{-1} \int_{A_{b n d y}^{n}} L^{n}[1-\operatorname{sign}(\hat{\mathbf{s}} d \boldsymbol{A})]\left(I^{+}-I^{-}\right) \hat{\mathbf{s}} d \boldsymbol{A} \\
&= \frac{1}{2}\left(M^{n}\right)^{-1} \int_{A_{b n d y}^{n}} L^{n}[1-\operatorname{sign}(\hat{\mathbf{s}} d \boldsymbol{A})]\left(0-I^{-}\right) \hat{\mathbf{s}} d \boldsymbol{A} \\
& \quad+\frac{1}{2}\left(M^{n}\right)^{-1} \int_{A_{b n d y}^{n}} L^{n}[1-\operatorname{sign}(\hat{\mathbf{s}} d \boldsymbol{A})]\left(I^{+}-0\right) \hat{\mathbf{s}} d \boldsymbol{A}
\end{aligned}
$$

Then, we shift the second part to the RHS of Eq. (21) and treat it as a source term. If scattering and/or reflecting and emissive walls are present, radiative intensities of different direction affect each other and therefore coupling of each direction is required. In the RTE solver, their contribution was treated as a source term using the most recently available intensity solutions, and numerical convergence was examined.

\subsection{Matrix-Free Formulation}

The matrix construction was found to consume a significant amount of computational resource and time. It was also difficult to implement it efficiently with GPGPUs. The solution adopted in this study consists of writing the algebraic relations of Eq. (21) for each wavenumber into the classic form

$$
\mathbf{M x}=\mathbf{b}
$$

where $\mathbf{M}$ is a $\left(N \times N_{p}\right) \times\left(N \times N_{p}\right)$ matrix, $\mathbf{x}=\left[\left(I_{m}^{n}\right)_{1 \leq n \leq N, 1 \leq m \leq N_{p}}\right]^{T}$ is the radiative intensity vector to be solved, and $\mathbf{b}$ corresponds to contribution from blackbody emission, i.e., the RHS term of Eq. (21) and in-scattering if applicable. Both $\mathbf{x}$ and $\mathbf{b}$ are vectors of size $N \times N_{p}$. Krylov subspace iteration methods do not require one to build the matrix $\mathbf{M}$ explicitly [37]. The ultimate goal of constructing a matrix is for the matrix-vector product computation in Krylov subspace iteration algorithms. By correlating Eq. (21) and Eq. (24), the matrix-vector product operation is identical to Eq. (21)'s LHS. This matrix-vector product can be evaluated using a traditional function in the computation framework of the DG method. With an additional vector-vector product function, we were able to implement a matrix-free RTE solver using the bi-conjugate gradient stabilized (Bi-CGSTAB) method described in [36]. Based on our computational studies of a variety of radiative problems and grids, the Bi-CGSTAB method with preconditioner was always able to satisfactorily produce converged solutions for matrix inversions.

\subsection{Preconditioner}

The preconditioner is of equal importance for the iterative method in matrix inversion. The condition number of matrix involved in the RTE solver increases significantly with DG order and grid refinements. Figure 1 shows the nonzero elements of the matrix (represented by a + sign) for a problem of $N=86$ tetrahedron elements 


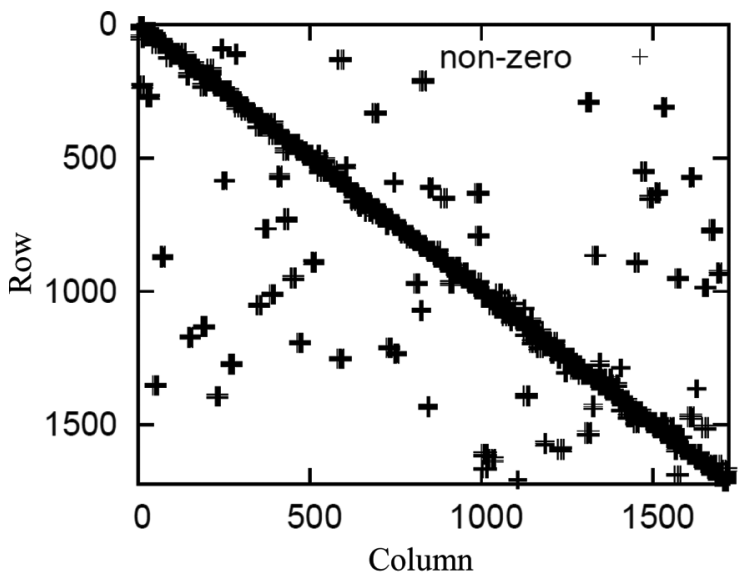

(a)

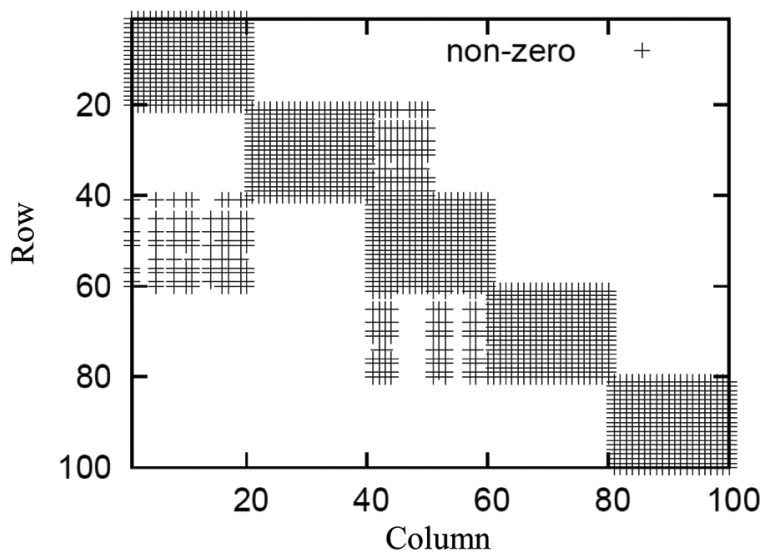

(b)

Figure 1. Nonzero elements for the $\left(N \times N_{p}\right) \times\left(N \times N_{p}\right)$ matrix of a radiative transfer problem with P-3 DG scheme $\left(N_{p}=20\right)$. The grid mesh consists of $N=86$ tetrahedron elements. (a) Full matrix. (b) Close-up view of the matrix.

with P-3 DG method $\left(N_{p}=20\right)$. A block diagonal pattern is clearly distinguishable. Then, Eq. (21) can be rewritten as

$$
\begin{aligned}
\left(M^{n}\right)^{-1} \int_{V^{n}} L^{n} \hat{\mathbf{s}} \cdot \nabla I^{n} d V+\left(M^{n}\right)^{-1} \int_{V^{n}} L^{n} \kappa I^{n} d V \\
-\frac{1}{2}\left(M^{n}\right)^{-1} \int_{A^{n}} L^{n}[1-\operatorname{sign}(\hat{\mathbf{s}} \cdot d \boldsymbol{A})] I^{-} \hat{\mathbf{s}} d \boldsymbol{A} \\
\quad+\frac{1}{2}\left(M^{n}\right)^{-1} \int_{A^{n}} L^{n}[1-\operatorname{sign}(\hat{\mathbf{s}} \cdot d \boldsymbol{A})] I^{+} \hat{\mathbf{s}} d \boldsymbol{A} \\
=\left(M^{n}\right)^{-1} \int_{V^{n}} L^{n} \kappa I_{b} d V
\end{aligned}
$$


The first three terms on the LHS of Eq. (25) contribute to the diagonal block, while the fourth term contributes to off-diagonal-block elements and the source terms [Eq. (23)] for the internal and external boundaries, respectively. Then, a block Jacobi preconditioner is the natural choice [38]. It depends on direction and the gas absorption coefficient in the second term of Eq. (25). Since most radiation transfer problems are convection-dominated, we neglected the second term, constructed a gas radiation model independent of preconditioner, and called it the partial block Jacobi preconditioner.

Finally, the computational kernels for the GPU were implemented using OpenCL [39], which provides portability across multiple computing platforms. To efficiently exploit the GPU's enormous capability of floating-point operations, computational tasks should be parallelizable. Most of the computation operators in the DG framework are carried out in an element-local manner. Therefore the RTE solver based on the GPU method is well suited for GPU computing [7]. For the GPU kernels of the RTE solver, we typically employed a thread block to carry out element-wise calculations, whereas the threads within the same block cooperate efficiently via shared memory. In the GPU computation mode, the entire solution process was performed on the GPU, with the exception of initialization (e.g., problem setup and grid reading and partitioning) and communication of fields on processor boundaries, which were performed by the CPU.

\section{RESULTS AND DISCUSSION}

The RTE solver developed in this study was implemented as a 3-D solver employing tetrahedron elements. It can also be employed to investigate 2-D or $1-\mathrm{D}$ radiative problems which are frequently encountered in the literature [2]. To simulate 2-D problems (in the $x-y$ plane), a 3-D mesh with one cell in the $z$ direction was generated and the two $x-y$ planes were set to be specularly reflecting. It is evident that a specularly reflecting boundary results in direction coupling and increases computational needs/requirements. To diminish the coupling effect, we used a grid of tall cells with an aspect ratio of 50 in the $z$ direction, which was realized by scaling up a homogeneous grid in the $z$ direction. Based on our experience, 3 to 5 iterations were sufficient to ensure the convergence of direction coupling using tall cells.

\subsection{3-D Radiative Transfer in Nongray, Emitting, Absorbing, and Nonscattering Media with Black Boundaries Exposed to Diffuse Irradiation}

Let us consider a parallelepiped enclosure with the dimension of $[-1.0 \mathrm{~m}$, $1.0 \mathrm{~m}] \times[-1.0 \mathrm{~m}, 1.0 \mathrm{~m}] \times[0.0 \mathrm{~m}, 4.0 \mathrm{~m}]$ in the $x, y$, and $z$ directions, respectively. All six of the enclosure's side walls were black at $300 \mathrm{~K}$. Temperature along the enclosure centerline $(x=0, y=0, z)$ was prescribed as

$$
T_{c}(0,0, z)= \begin{cases}T_{i}+\left(1,800-T_{i}\right) z / 0.375 & \text { for } 0 \leq z \leq 0.375 \mathrm{~m} \\ 1,800+\left(T_{e}-1,800.0\right)(z-0.375) /(4.0-0.375) & \text { for } z>0.375 \mathrm{~m}\end{cases}
$$


where $T_{i}=400 \mathrm{~K}$ and $T_{e}=800 \mathrm{~K}$. The gas temperature was symmetric about the centerline with

$$
T(r)=\left(T_{c}-T_{e}\right) f(r / R)+T_{e} \quad \text { with } f(r / R)=1-3(r / R)^{2}+2(r / R)^{3}
$$

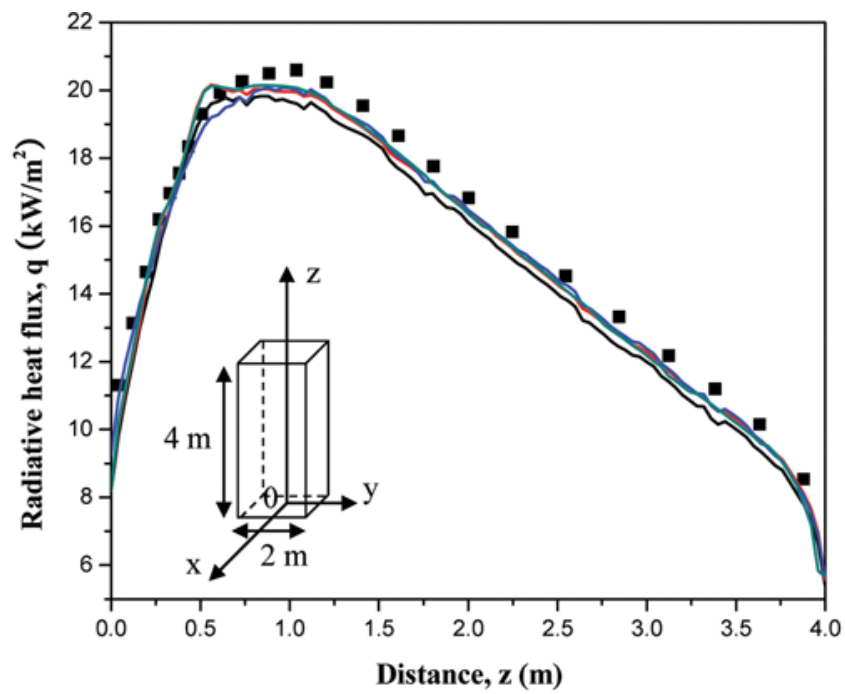

(a)

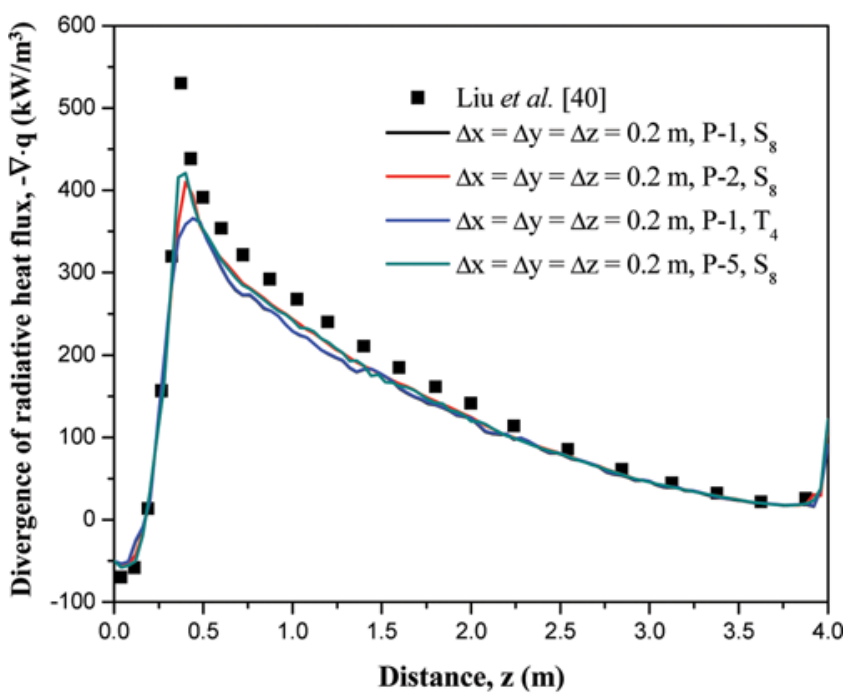

(b)

Figure 2. Comparison of simulated (a) radiative heat flux along the centerline of a sidewall $(x=-1.0$, $y=0, z)$ and $(b)$ divergence of radiative heat flux along the centerline $(x=0, y=0, z)$ for a 3-D rectangular enclosure containing absorbing, emitting, and nonscattering gas mixture at a specified temperature [Eq. (26)] with SNBCK gas model and different DG orders (P-1, P-2, and P-5), grid size $(\Delta x=\Delta y=\Delta z)$, and angular quadratures $\left(S_{8}\right.$ and $\left.T_{4}\right)$ with results reported in [40] (color figure available online). 
where $r=\sqrt{x^{2}+y^{2}}$ is the distance from the centerline and $R=1.0 \mathrm{~m}$. The gas mixture consisted on $\mathrm{CO}_{2}$ and water vapor in $\mathrm{N}_{2}$ with uniform mole fractions of $0.1,0.2$, and 0.7 , respectively. We performed simulations using (1) uniform grid resolutions $\Delta x=\Delta y=\Delta z=0.2 \mathrm{~m}$ corresponding to $10 \times 10 \times 20$ cells, (2) three different DG orders (P-1, P-2, or P-5), and (3) two different angular quadrature sets ( $S_{8}$ or $\left.T_{4}\right)$. Figure 2 shows the computed wall heat flux along the centerline of a side wall $(x=-1.0 \mathrm{~m}, y=0, z)$, with different simulation settings and the distribution of divergence of radiative heat flux along the centerline of the enclosure $(x=0, y=0, z)$. The predictions were compared with the results reported in [40], obtained using $T_{4}$ angular discretization and finite-volume spatial discretization with $17 \times 17 \times 24$ rectangular cells in the $x, y$, and $z$ directions. The average and maximum relative errors between our numerical results for the radiative heat flux along $(-1,0, z)$ and those reported in [40] were $5 \%$ and $12 \%$, respectively. In addition, the average and maximum relative errors between our numerical results for the divergence of radiative heat flux along $(0,0, z)$ and those reported in [40] were $12 \%$ and $19 \%$, respectively. Our predictions using different combinations of DG orders, grid resolutions, and angular quadrature types all agree well with those reported in [40].

This study demonstrates that in order to achieve accurate simulation results using the RTE solver, one can choose to employ coarse-grid resolution and higher order of DG scheme or fine-grid resolution and lower order of DG scheme. Based on our testing, the combination of higher-order DG scheme and coarser grid is preferable in terms of accuracy and computational time for problem with smooth solutions. However, finer grid resolution is required for areas with sharp discontinuities in the solution.

\subsection{1-D Radiative Transfer in Gray, Emitting, Absorbing, and Nonscattering Media with Reflecting Boundaries Exposed to Diffuse Irradiation}

All previous studies using the DG method to solve the RTE considered black walls [23, 26, 29]. This section discusses the validity of our RTE solver for problems involving nonblack diffusive walls and diffusely emitting and reflecting boundaries. The incoming radiative intensity from nonblack diffusive walls is determined by the reflected outgoing intensity and the blackbody intensity. Here, we used the benchmark problem solved analytically in [41]. It consists of a 1-D gas layer of thickness $L$ sandwiched between two walls at temperature $T_{w}=500 \mathrm{~K}$ with emissivity $\epsilon=0.5$. The gas was treated as gray, emitting, absorbing, and nonscattering, with optical thickness $\tau_{L}=\kappa L$ equal to $0.1,1.0$, and 10 . The gas temperature was uniform and equal to $T_{g}=1,500 \mathrm{~K}$. The walls were gray and diffusively emitting and reflecting. Figure 3 compares our numerical predictions with the exact solution [41] for the divergence of the dimensionless radiative heat flux as a function of $x / L$ defined as [41]

$$
\frac{d q^{*}}{d \tau}=\frac{d q / d \tau}{\sigma\left(T_{w}^{4}-T_{g}^{4}\right)}
$$




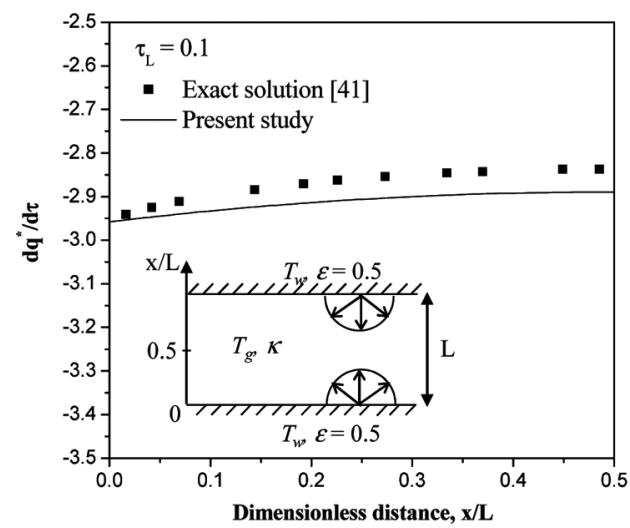

(a)

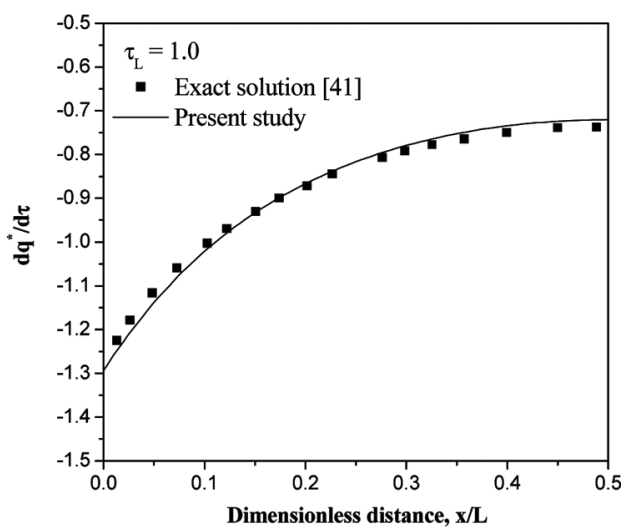

(b)

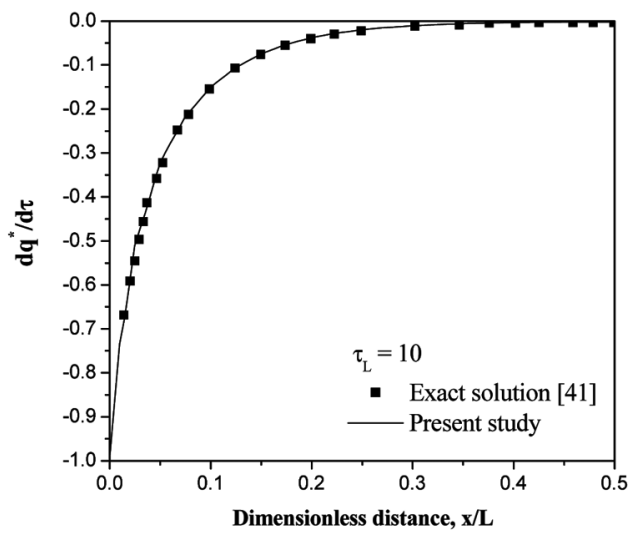

(c)

Figure 3. Comparison of divergence of dimensionless radiative heat flux [Eq. (2)] between DG method solution and the exact solution [41] for 1-D absorbing and emitting gray gas at $T_{g}=1,500 \mathrm{~K}$ with gray diffusely reflecting and emitting walls at $T_{w}=500 \mathrm{~K}$ with $\epsilon=0.5$. The medium optical thickness as were (a) $\tau_{L}=0.1$, (b) $\tau_{L}=1.0$, and $(c) \tau_{L}=10.0$.

The numerical results were obtained using the P-6 DG method and $S_{8}$ angular discretization with a grid consisting of 125 tetrahedron elements. They fell within $2 \%$ of the exact solution [41] for all optical thicknesses considered. These results demonstrate that the DG method can accurately simulate diffusely emitting and reflecting boundaries.

\subsection{2-D Radiative Transfer in Gray and Purely Scattering Media with Black Boundaries Exposed to Diffuse Irradiation}

This section explores the performance of the DG method to deal with different scattering phase functions. Kim and Lee [42] considered a 2-D $1 \mathrm{~m} \times 1 \mathrm{~m}$ square enclosure filled with a scattering medium and 5 different scattering phase functions, namely, (1) isotropic, (2) two forward with Henyey-Greenstein asymmetric factor 
$g=0.84534(\mathrm{~F} 1)$ and $0.66972(\mathrm{~F} 2)$, and (3) two backward with $g=-0.18841$ (B1) and -0.4 (B2) (see Figure 2 in [42]). Trivic et al. [43] simulated similar problems using the finite-volume method for angular discretization with $25 \times 25$ control volumes. Trivic and Amon [44] extended their 2-D study [43] to 3-D problem, with the same set of scattering phase functions.

In the present study, the medium was purely scattering and all walls were black and cold except the bottom wall at $(x, y=0)$ which had blackbody emissive power $E_{b w}$. In addition, the dimensionless fluence rate $G^{*}$ was defined as $G^{*}=G / 4 E_{b w}$ and the dimensionless radiative heat flux was defined as $q^{*}=q / E_{b w}$ [42]. The simulations were carried out using P-1 DG method on a grid consisting of 4,810 tetrahedron elements and using the finite-volume method for angular discretization with $N_{\theta}=4$ and $N_{\phi}=60$. Figures $4 \mathrm{a}$ and $4 \mathrm{~b}$ show the dimensionless (a) heat flux $q^{*}$ and (b) fluence rate $G^{*}$ predicted as a function of distance $y$ along the centerline $x=0.5 \mathrm{~m}$. The average and maximum relative errors between our numerical results for the dimensionless heat flux $q^{*}(0.5, y)$ and those reported in $[42,43]$ were $3 \%$ and $4 \%$, respectively. In addition, the average and maximum relative errors between our numerical results for dimensionless fluence rate $G^{*}(0.5, y)$ and those reported in [42, 43 ] were $1 \%$ and $4 \%$, respectively. Overall, the numerical predictions were in good agreement with the data obtained from $[42,43]$ for all scattering phase functions considered.

\subsection{3-D Radiative Transfer in Gray, Emitting, Absorbing, and Scattering Media with Black and Reflecting Boundaries Exposed to Diffuse Irradiation}

This section compares our simulation results for the 3-D combustor shown in Figure 5 with those provided in $[45,46]$. The geometric feature of this problem was quite complex and grid generation was challenging, as there were five thin baffles located at a corner of the domain. A in-house grid-generation tool was used to generate an unstructured body-fitted grid. The medium was gray, emitting, absorbing, and scattering, with the following temperature and absorption coefficient spatial distributions:

$$
\begin{cases}\kappa=0.20 \mathrm{~m}^{-1}, T=1,600 \mathrm{~K} & \text { for } z \leq 5 \mathrm{~m} \\ \kappa=0.25 \mathrm{~m}^{-1}, T=2,000 \mathrm{~K} & \text { for } 5 \leq z \leq 10 \mathrm{~m} \\ \kappa=0.20 \mathrm{~m}^{-1}, T=1,600 \mathrm{~K} & \text { for } 10 \leq z \leq 20 \mathrm{~m} \\ \kappa=0.18 \mathrm{~m}^{-1}, T=1,200 \mathrm{~K} & \text { for } 20 \leq z \leq 30 \mathrm{~m}\end{cases}
$$

Temperature and emissivity of the boundary region located at $x=10 \mathrm{~m}$ and $22 \mathrm{~m}$ $\leq z \leq 30 \mathrm{~m}$ were specified to be $1,200 \mathrm{~K}$ and 1.0 , respectively. The temperature and emissivity at other boundaries, including at the baffle surfaces, were specified to be $800 \mathrm{~K}$ and 0.65 , respectively. Two cases with different uniform scattering albedos, $\omega=0.0$ and 0.5 , were simulated. DOM with $S_{8}$ angular discretization scheme and P-3 DG method were employed. The grid consisted of 54,266 tetrahedral elements. In [45, 46], 72,000 hexahedral elements were employed with a block-off-region procedure to deal with the irregular geometric domain, i.e, nonorthogonal walls were represented with a staircase grid. 


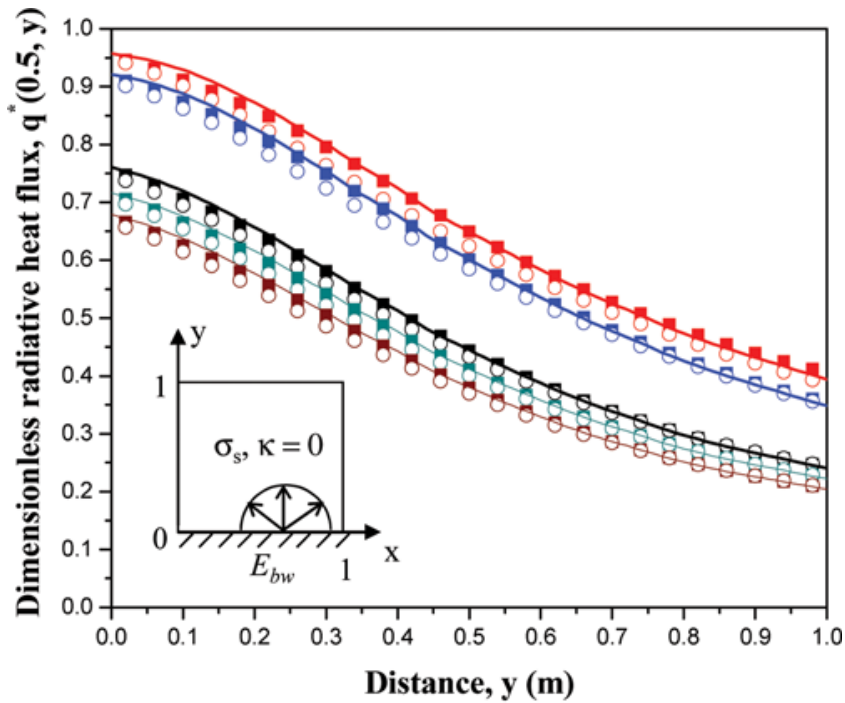

(a)

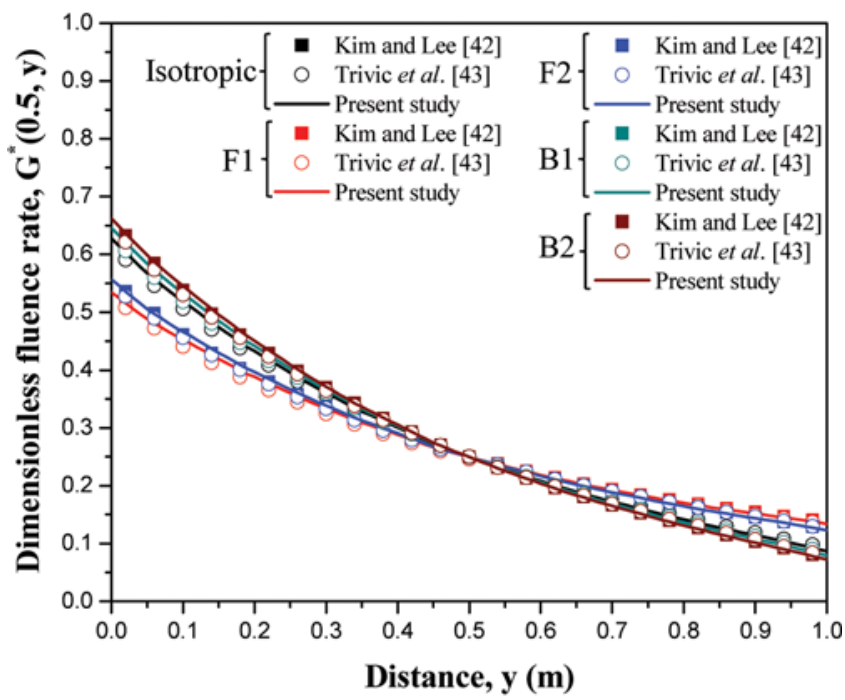

(b)

Figure 4. (a) Dimensionless heat flux $q^{*}(0.5, y)$ and $(b)$ fluence rate $G^{*}(0.5, y)$ along the $y$-axis for center of $x$-axis in a 2-D enclosure containing purely scattering media with isotropic, strongly forward (F1 and F2), and strongly backward (B1 and B2) scattering phase functions. Our results are compared with previously reported studies $[42,43]$ obtained using finite-volume method and DOM. Here, $q^{*}(x, y)=q(x, y) / E_{b w}$ and $G^{*}(x, y)=G(x, y) / 4 E_{b w}$ (color figure available online).

Figure 5 shows the net radiative heat flux on the front wall, side wall, and back wall for $(a) \omega=0.0$ and $(b) \omega=0.5$ of the 3 -D combustor. Discrepancies in flux contour can be observed around the concave area compared with the solutions reported in Figure 10 of [45] and Figure 9 of [46]. We believe that our simulation produces 

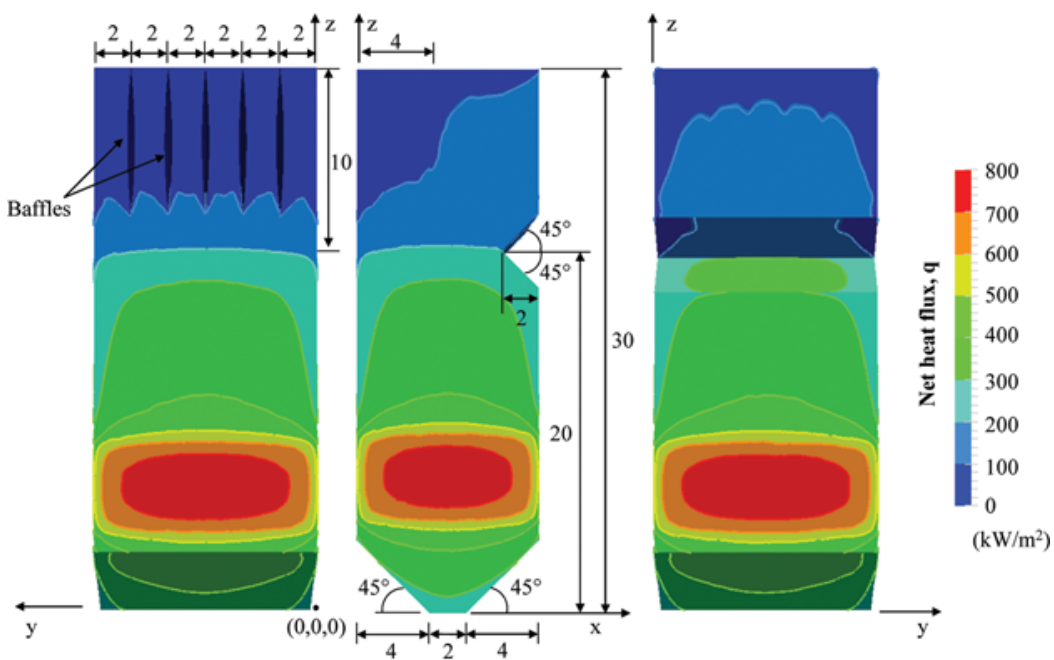

(a)

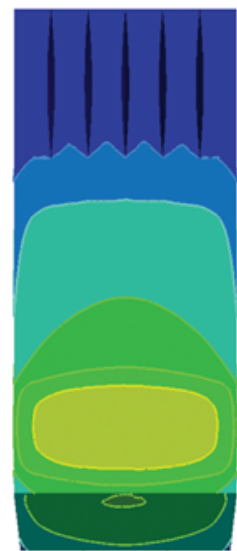

Front wall $(\mathrm{x}=0 \mathrm{~m})$

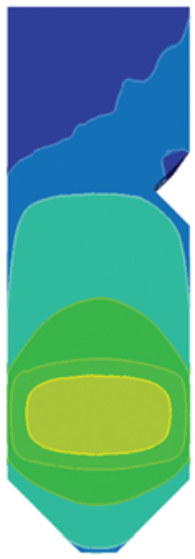

Side wall

(b)

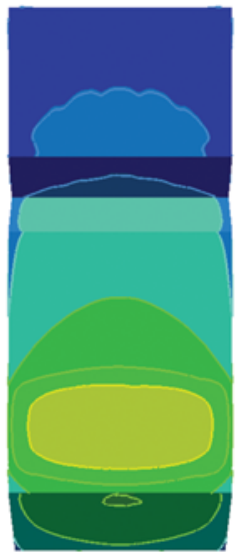

Back wall $(\mathrm{x}=10 \mathrm{~m})$

Figure 5. Geometry, dimension, and simulation results of the net radiative heat flux on the front, side, and back walls of a 3-D combustor chamber [46] with absorbing, emitting, $(a)$ nonscattering medium $(\omega=0.0)$ and $(b)$ scattering medium $(\omega=0.5)$. All dimensions are in meters (color figure available online).

better results than that of $[45,46]$ in these areas since we employed a boundaryconforming mesh, instead of block-out technique, on a Cartesian grid to represent the nonorthogonal boundaries. As pointed out in [47], the block-off technique introduced significant error in the computed heat flux on nonorthogonal walls.

\subsection{2-D Radiative Transfer in Gray Absorbing and Scattering Media with Black Boundaries Exposed to Collimated Irradiation}

This section discusses validation of our RTE solver for applications with collimated incident irradiation. Figure $6 a$ shows the cylindrical enclosure of radius $R$ 


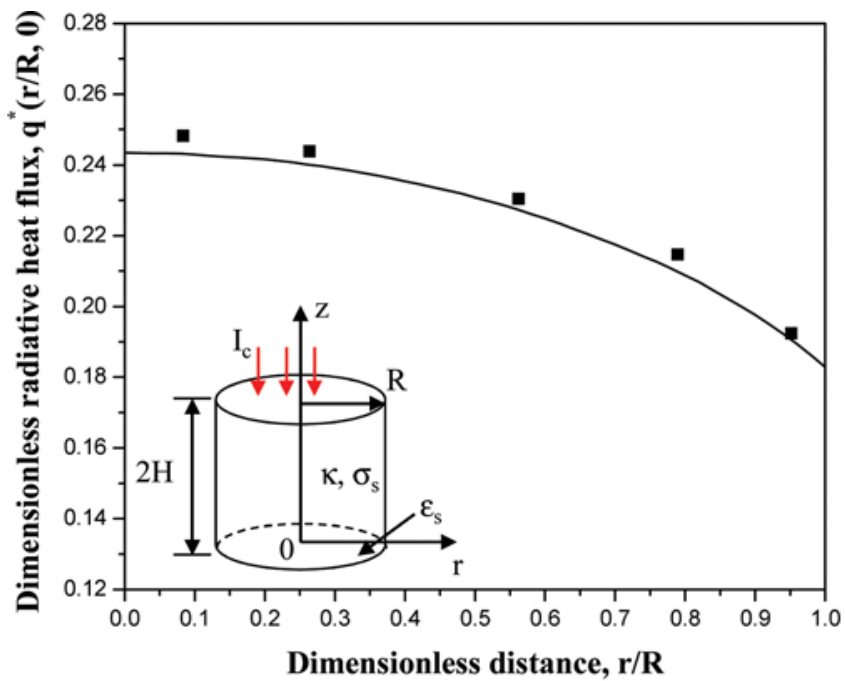

(a)

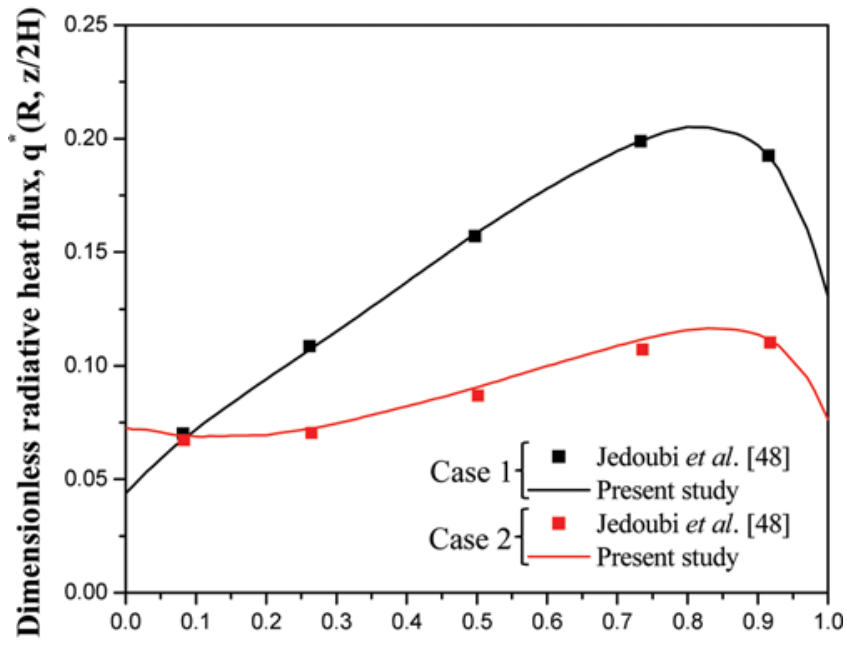

Dimensionless distance, $\mathbf{z} / \mathbf{2 H}$

(b)

Figure 6. Dimensionless radiative heat flux along $(a)$ the bottom wall $q^{*}(r / R, 0)$ and $(b)$ the sidewall of the cylindrical enclosure $q^{*}(R, z / 2 H)$ with $R=H=1 \mathrm{~m}$ containing cold, absorbing, and isotropically scattering medium exposed to collimated radiation incident from the top wall. Case 1 is such that $\epsilon_{s}=1.0$, $\kappa=0.0 \mathrm{~m}^{-1}$, and $\sigma_{s}=1.0 \mathrm{~m}^{-1}$. Case 2 is such that $\epsilon_{s}=0.5, \kappa=0.3 \mathrm{~m}^{-1}$, and $\sigma_{s}=0.7 \mathrm{~m}^{-1}$ [48] (color figure available online).

and height $H$ such that $R=H=1.0 \mathrm{~m}$. Normal collimated radiation $\left(I_{c}=1.0 \mathrm{~W} / \mathrm{m}^{2} \mathrm{sr}\right)$ was incoming from the top wall. The enclosure was filled with cold, gray, and homogeneous absorbing and isotropically scattering medium with optical radius $\tau_{R}=(\kappa+$ $\left.\sigma_{s}\right) R=1.0$. The cylindrical side wall was cold and black $\left(\epsilon_{s}=1.0, T_{s}=0 \mathrm{~K}\right)$. Jendoubi 
et al. [48] studied two cases. Case 1 had bottom wall emissivity $\epsilon_{s}=1.0$ while the medium absorption and scattering coefficients were $\kappa=0.0 \mathrm{~m}^{-1}$ and $\sigma_{s}=1.0 \mathrm{~m}^{-1}$, respectively. Case 2 had bottom wall emissivity $\epsilon_{s}=0.5$ and absorption and scattering coefficients $\kappa=0.3 \mathrm{~m}^{-1}$ and $\sigma_{s}=0.7 \mathrm{~m}^{-1}$, respectively. Angular discretization scheme $T_{4}$ and P-3 DG method were employed. The grid consisted of 6,872 tetrahedral elements. The dimensionless radiative heat flux was defined as $q^{*}(r, z)=q(r, z) / E_{b}$, where $E_{b}$ is the blackbody emissive power, set at $E_{b}=\pi$. Figure 6 compares the numerical predictions for the dimensionless radiative heat flux $(a)$ at the bottom wall $q^{*}(r / H, z)$ and along the cylinder side wall $q^{*}(z / 2 H, z)$ with those reported in [48] for Cases 1 and 2. The average and maximum relative errors between them were $3 \%$ and $4 \%$, respectively. This demonstration that the DG method can also simulate collimated irradiation problems.

\section{SPEED-UP OF GPU VERSUS CPU}

Let us consider a benchmark problem consisting of a 3-D square enclosure $2 \mathrm{~m} \times 2 \mathrm{~m} \times 2 \mathrm{~m}$ having cold black walls and containing a gray, absorbing, emitting, and nonscattering medium with the following spatial distributions of the blackbody radiation intensity:

$$
\begin{aligned}
I_{b}(x, y, z)= & \frac{\pi}{2 \kappa} s_{x} \cos \left(\frac{\pi}{2} x\right)\left[1+\sin \left(\frac{\pi}{2} y\right)\right]\left[1+\sin \left(\frac{\pi}{2} z\right)\right] \\
& +\frac{\pi}{2 \kappa} s_{y}\left[1+\sin \left(\frac{\pi}{2} x\right)\right] \cos \left(\frac{\pi}{2} y\right)\left[1+\sin \left(\frac{\pi}{2} z\right)\right] \\
& +\frac{\pi}{2 \kappa} s_{z}\left[1+\sin \left(\frac{\pi}{2} x\right)\right]\left[1+\sin \left(\frac{\pi}{2} y\right)\right] \cos \left(\frac{\pi}{2} z\right) \\
& +\left[1+\sin \left(\frac{\pi}{2} x\right)\right]\left[1+\sin \left(\frac{\pi}{2} y\right)\right]\left[1+\sin \left(\frac{\pi}{2} z\right)\right]
\end{aligned}
$$

where the absorption coefficient was $\kappa=0.1 \mathrm{~m}^{-1}$ and a single angular direction, $\hat{\mathbf{s}}=\left(s_{x}, s_{y}, s_{z}\right)=(0.62402,0.52802,0.57602)$ was considered. The temperature field can be defined as $T(x, y, z)=\left[\pi I_{b}(x, y, z) / \sigma\right]^{1 / 4}$, where $\sigma\left(=5.67 \times 10^{-8} \mathrm{~W} / \mathrm{m}^{2} \cdot \mathrm{K}^{4}\right)$ is the Stefan-Boltzmann constant. The RTE was solved using the DG method with order ranging from P-1 to P-4 and with two different grids consisting of 2,861 and 22,655 tetrahedron elements. The CPU code was executed on a workstation with Intel Xeon CPU E5620, $2.40 \mathrm{GHz}$. The GPU code was executed on a NVIDIA ${ }^{\circledR}$ GeForce ${ }^{\circledR}$ GTX 480 card with OpenCL 1.0 CUDA 3.2.1. The computational time for the solution along one radiative angular direction were used to calculate the speed-up associated with using GPU computing. Figure 7 shows the speed-up of the RTE solver in GPU mode versus CPU mode for a benchmark problem. The speed-up was up to 4.0 and increased with increasing DG scheme order. Indeed, higher-order DG requires more floating points and is better suited to GPU computing.

\section{INTEGRATION OF RTE SOLVER WITH CFD DATA AND SOLVERS}

The RTE solver can also be coupled with other physical solvers. Generally, the grids employed by these solvers are different from that used by the RTE solver. 


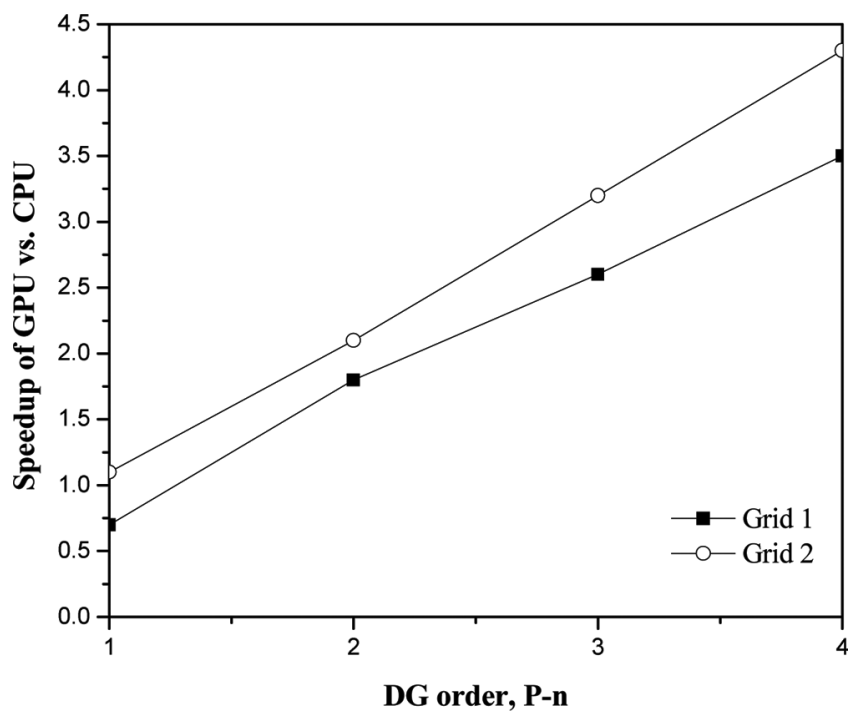

Figure 7. Speed-up of GPU versus CPU implementations as a function of DG order for a 3-D radiative transfer problem in gray, absorbing, emitting, and nonscattering medium at the prescribed temperature field with $\kappa=0.1 \mathrm{~m}^{-1}$. Grid 1 and grid 2 consisted of 2,861 and 22,655 tetrahedron elements, respectively.

Variables interpolation between different grids is therefore the primary challenge. In this section, we present a case study of rocket plume radiation simulation using the flow fields computed using a computational fluid dynamics solver described in [49].

\subsection{CFD Simulation on Atlas II Rocket Plume}

A very versatile 3-D flow solver was used for solving aerodynamics and aerothermodynamics problems governed by the Euler or Reynolds-averaged Navier-Stokes (RANS) equations [49-53]. The discretization was based on a total variation diminishing (TVD) [54-56] formulation for the inviscid fluxes and second-order central differencing for the viscous fluxes using a finite-volume framework. The TVD formulation allowed the CFD solver to automatically handle flow discontinuities without any additional dissipation operators [57]. The Menter one-equation turbulence model [58] was used in the CFD solver. We accounted for 9 gas species, $\mathrm{H}_{2}, \mathrm{O}_{2}, \mathrm{H}_{2} \mathrm{O}, \mathrm{OH}, \mathrm{O}, \mathrm{H}, \mathrm{CO}, \mathrm{CO}_{2}$, and $\mathrm{N}_{2}$, and considered 12 reactions steps. The CFD solver used a second-order Rusanov scheme with $25 \%$ of the numerical dissipation added and standard minmod slope limiting with compression factor 1.25 [49]. The CFD solver can be used for a wide variety of situations, including (1) unsteady and steady flows, (2) low-speed, subsonic, transonic, supersonic, and hypersonic flows, (3) perfect gas, equilibrium air curve-fit, frozen, equilibrium, and finite-rate chemistry, (4) viscous and inviscid flows, (5) simple and complex geometries, and (6) internal and external flows. Aeroheating may be modeled by a variety of boundary conditions including (i) constant-temperature walls, (ii) variabletemperature walls, (iii) radiative equilibrium walls, and (iv) catalytic walls. 


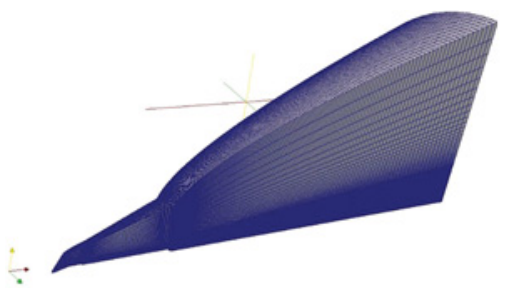

(a)

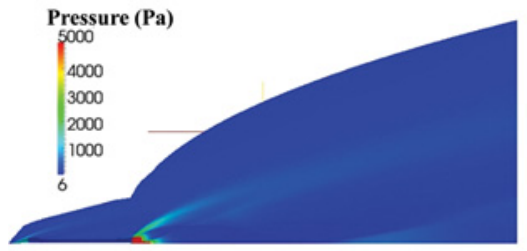

(c)

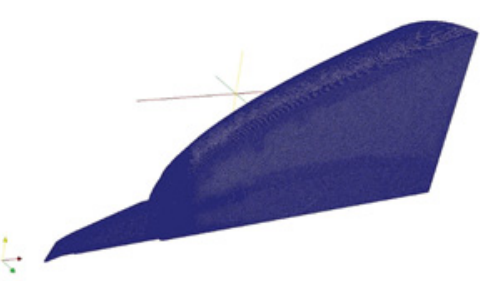

(b)

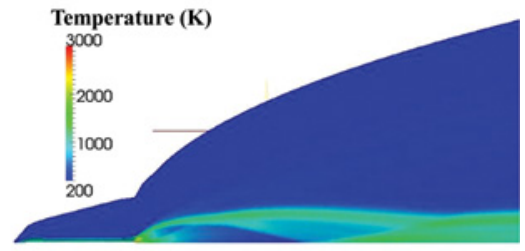

(d)

Figure 8. Grids consisting of (a) 2,849,552 hexahedron elements for the CFD solver, (b) 1,896,071 tetrahedron elements for the RTE solver, $(c)$ interpolated pressure fields, and $(d)$ interpolated temperature fields (color figure available online).

The geometry of the Atlas II rocket for mesh generations was based on that employed by Alexeenko et al. [6]. Due to the symmetry of the problem, a quarter of the physical domain was discretized and the appropriate symmetric boundary conditions were used. Different grids for CFD and the RTE solvers were generated, as shown in Figures $8 a$ and $8 b$. The CFD grid consisted of 2,849,552 hexahedron elements, whereas the RTE solver used a grid with 1,896,071 tetrahedron elements. Flow and boundary conditions for the case of $40-\mathrm{km}$ altitude presented in [6] were adopted for the CFD solver. The finite rate chemistry employed by the CFD solver can be found in [59].

\subsection{Interpolation of Flow Fields}

First, the CFD solver computed the flow, pressure, species, and temperature fields. These field solutions were transferred to the RTE solver by interpolating the hexahedron-based CFD grid to the tetrahedron-based RTE grid. To facilitate the interpolation of flow field solutions from the CFD grid to the RTE grid, we constructed a kd-tree [60] consisting of all CFD grid vertices. The kd-tree data structure has been widely employed for efficient nearest-neighbor search on points cloud [60]. The flow variables at any spatial location can be interpolated from the values of its nearest-neighbor vertices of the CFD grid.

\subsection{Radiative Computation of Plume Field}

Alexeenko et al. [6] performed plume radiation signature calculations using (1) the standard infrared radiation model (SIRRM) [61], (2) the standard plume 
untraviolet code (SPURC) [62], and (3) the atmospheric radiative transfer code (MODTRAN) [63] for atmospheric attenuation. A series of results on the infrared spectral band at $4.667-4.802 \mu \mathrm{m}$ was presented in [6]. In this study, we verified the plume radiance calculation with the present RTE solver. Finite-volume angular discretization with the resolution of $20 \times 9$ for $\phi$ and $\theta$ were employed. The hybrid SNBCK gas model of our RTE solver was used over a spectral region $2,100-2,250 \mathrm{~cm}^{-1}$, corresponding to $4.444-4.762 \mu \mathrm{m}$. We compared the radiation intensity at this band with that of $4.667-4.802 \mu \mathrm{m}$ in [6]. The narrow-band gas model database with our RTE solver was for flow condition at $1 \mathrm{~atm}$ by default, whereas the pressure of the plume field at $40-\mathrm{km}$ altitude is fairly low [6]. Thus, the absorption coefficient was scaled from the hybrid SNBCK gas model condition with a ratio of $P / P_{\text {atm }}$, in which the pressure field $P$ was computed from the CFD solver [64].

Figure $9 b$ shows the calculated radiance in the $2,100-2,250 \mathrm{~cm}^{-1}$ band at the observation angles of $90^{\circ}$ and $81^{\circ}$ for aspect angle $\varphi$ and roll angle $\theta$, respectively, as defined in Figure $9 a$. The magnitude and pattern of the plume radiance are qualitatively consistent with that presented in [6].

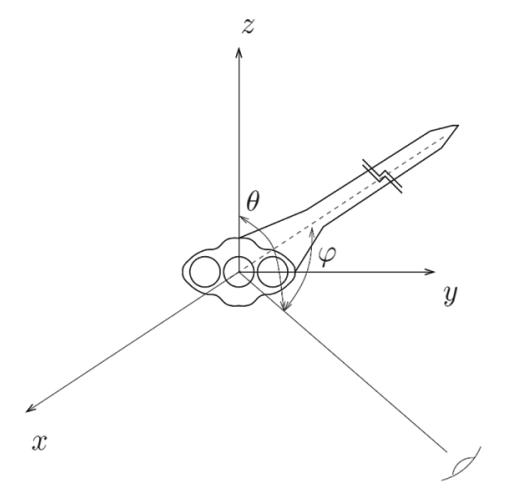

Observer

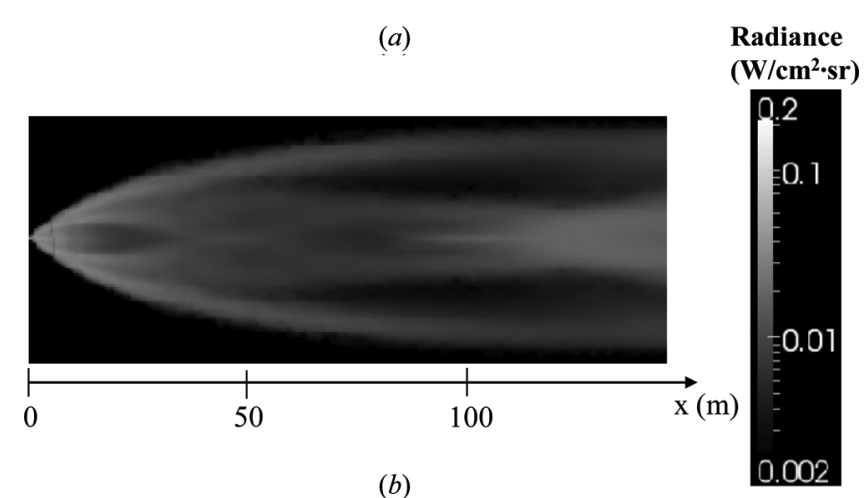

Figure 9. (a) Definition of aspect angle $(\varphi)$ and roll angle $(\theta)$ of observation. (b) Computed plume radiance $\left[\mathrm{W} / \mathrm{cm}^{2} \cdot \mathrm{sr}\right]$ in the spectral band of $4.444-7.762 \mu \mathrm{m}$ at observing angles of $\varphi=90^{\circ}$ and $\theta=81^{\circ}$. 


\subsection{Performance of Parallel CPU and GPU Computations}

In this section, simulations of the Atlas II rocket plume were performed using two RTE grids consisting of 1,896,071 and 181,213 tetrahedron elements. A series of studies to check the scalability of the RTE solver with respect to parallel computing was carried out. Simulations were performed on the CPU and GPU clusters of Brown University Center for Computation \& Visualization (CCV). The CPU cluster consisted of 2168 -core nodes, and the GPU cluster consisted of 44 8-core nodes with 2 NVIDIA Tesla M2050 cards installed in each node. We recorded the computational time taken by each processor with our RTE solver in solving the Atlas II rocket radiation transfer problem at a single angular direction and band quadrature.

CPU parallel solutions of the RTE were carried out using P-1 DG method with a fine-grid mesh of 1,896,071 elements. Here, the number of nodes as well as the number of cores of each node employed need to be specified. Table 1 lists the CPU time for each processor for computation using 2, 4, and 8 cores for each node. It demonstrated good speed-up with increasing number of processors. The speed-up efficiency was around $95 \%$ and increased as the number of cores decreased. By comparing the CPU time in Table 1 for cases with the same total number of processors, computations always ran faster when fewer cores were used in each node. This discrepancy may be attributed to the memory bus saturation when more cores of each node are involved in computations.

Parallel computations with CPU and GPU for solving RTE were performed using the P-3 DG method for a coarse grid consisting of only 181,213 elements. Based on our experience, the simulation results for the coarse grid with P-3 DG method should be more accurate than those obtained with the P-1 DG method and a finer grid (1,896,071 elements). Since each node of the Brown CCV GPU cluster had only 2 NVIDIA Tesla cards, we used 2 processing units of each node in GPU as well as CPU parallel computations for fair comparison. Table 2 summarizes the computational time for each processor for the CPU and GPU computations. The GPU computations demonstrated a consistent speed-up by a factor of 3 over the CPU computations. The parallel scalability could not be sustained to the extent observed in the fine-grid computations (Table 1). With smaller grid size, the computational tasks could not keep all the processors busy as the number of processors

Table 1. CPU times for each processor using the 2, 4, and 8 cores of each node required to solve the RTE with P-1 DG scheme and a grid consisting of 1,896,071 tetrahedral elements

\begin{tabular}{lccc}
\hline No. of cores & No. of processors & Node $\times$ core & Time (s) \\
\hline 2 & 8 & $4 \times 2$ & 78.72 \\
& 16 & $8 \times 2$ & 39.43 \\
4 & 32 & $16 \times 2$ & 19.32 \\
& 16 & $4 \times 4$ & 43.20 \\
8 & 32 & $8 \times 4$ & 21.28 \\
& 64 & $16 \times 4$ & 11.00 \\
& 32 & $8 \times 8$ & 26.47 \\
& 64 & $16 \times 8$ & 13.80 \\
& 128 & & 6.95 \\
\hline
\end{tabular}


Table 2. CPU times for each processor using 2 processing units (CPU or GPU) of each nodes and P-3 DG scheme with the grid consisting of 181,213 elements

\begin{tabular}{lccr}
\hline & processing & \multicolumn{2}{c}{ Time $(\mathrm{s})$} \\
\cline { 3 - 4 } No. of processors & Node $\times$ unit & CPU & GPU \\
\hline 8 & $4 \times 2$ & 51.36 & 15.41 \\
16 & $8 \times 2$ & 27.48 & 8.08 \\
32 & $16 \times 2$ & 15.90 & 5.05 \\
\hline
\end{tabular}

increased beyond a certain level. Comparing the CPU computational times in Table 2 with those in Table 1, the P-3 coarse-grid computations run faster than the corresponding P-1 fine-grid cases. This comparison demonstrates, again, the advantage of high-order numerical schemes.

\section{CONCLUSION}

This study presented a high-order discontinuous Galerkin (DG), combined with finite-volume (FVM) and discrete-ordinates method (DOM) for solving the RTE. The DG method was incorporated with nongray gas radiation models such as spectral line-based weighted-sum-of-gray-gases (SLW) and hybrid SNBCK model. Both diffusively and specularly reflecting walls were modeled along with diffuse and collimated incident irradiation in 3-D enclosures. A case study in modeling the radiance emitted by a rocket plume was presented. An important aspect of this work is the porting of the DG RTE solver to GPUs, demonstrating very attractive computational speed-up. Studies have revealed which computations benefit the most from GPU acceleration. The CPU/GPU parallel high-order-accurate solver bears the potential for high computational efficiency in large-scale RTE simulations with extensive variations in optical thickness. Further improvements in hp-adaptive solution procedures are currently being pursued.

\section{REFERENCES}

1. M. P. Mengüç and R. Viskanta, Radiative-Transfer in 3-Dimensional Rectangular Enclosures Containing Inhomogeneous, Anisotropically Scattering Media, J. Quant. Spectrosc. Radiat. Transfer, vol. 33, pp. 533-549, 1985.

2. M. F. Modest, Radiative Heat Transfer, 2nd ed., Academic Press, San Diego, CA, 2003.

3. R. Viskanta, Computation of Radiative Transfer in Combustion Systems, Int. J. Numer. Meth. Heat Fluid Flow, vol. 18, pp. 415-442, 2008.

4. R. Viskanta and M. P. Mengüç, Radiation Heat Transfer in Combustion Systems, Prog. Energy Combustion Sci., vol. 13, pp. 97-160, 1987.

5. R. M. Huffaker, Current Research on Infrared Radiation from Rocket Exhaust, J. Quant. Spectrosc. Radiat. Transfer, vol. 8, pp. 87-104, 1968.

6. A. A. Alexeenko, N. E. Gimelshein, D. A. Levin, R. J. Collins, R. Rao, G. V. Candler, S. F. Gimelshein, J. D. Hong, and T. Schilling, Modeling of Flow, and Radiation in the Atlas Plume, AIAA J. Thermophys. Heat Transfer, vol. 16, pp. 50-57, 2002.

7. A. Klockner, T. Warburton, J. Bridge, and J. S. Hesthaven, Nodal Discontinuous Galerkin Methods on Graphics Processors, J. Comput. Phys., vol. 228, pp. 7863-7882, 2009. 
8. W. F. Herget and J. D. Brasher, Remote Measurement of Gaseous Pollutant Concentrations Using a Mobile Fourier Transform Interferometer System, Appl. Opt., vol. 18, pp. 3404-3420, 1979.

9. N. Getoff, Control of Greenhouse Gases Emission by Radiation-Induced Formation of Useful Products. Utilization of $\mathrm{CO}_{2}$, Radiat. Phys. Chem., vol. 75, pp. 514-523, 2006.

10. L. Pilon, H. Berberoğlu, and R. Kandilian, Radiation Transfer in Photobiological Carbon Dioxide Fixation and Fuel Production by Microalgae, J. Quant. Spectrosc. Radiat. Transfer, vol. 112, pp. 2639-2660, 2011.

11. K. N. Liou, An Introduction to Atmospheric Radiation, 2nd ed., Academic Press, San Diego, CA, 2002.

12. M. K. Denison and B. W. Webb, A Spectral Line-Based Weighted-Sum-of-Gray-Gases Model for Arbitrary RTE Solvers, ASME J. Heat Transfer, vol. 115, pp. 1004-1012, 1993.

13. M. K. Denison and B. W. Webb, An Absorption-Line Blackbody Distribution Function for Efficient Calculation of Total Gas Radiative Transfer, J. Quant. Spectrosc. Radiat. Transfer, vol. 50, pp. 499-510, 1993.

14. M. K. Denison and B. W. Webb, Development and Application of an Absorption-Line Blackbody Distribution Function for $\mathrm{CO}_{2}$, Int. J. Heat Mass Transfer, vol. 38, pp. 1813-1821, 1995.

15. M. K. Denison and B. W. Webb, The Spectral Line-Based Weighted Sum of Gray Gases Model in Nonisothermal Nonhomogeneous Media, ASME J. Heat Transfer, vol. 117, pp. 359-365, 1995.

16. L. S. Rothman, I. E. Gordon, A. Barbe, D. Chris Benner, et al., The HITRAN 2008 Molecular Spectroscopic Database, J. Quant. Spectrosc. Radiat. Transfer, vol. 110, pp. 533-572, 2009.

17. V. P. Solovjov and B. W. Webb, SLW Modeling of Radiative Transfer in Multicomponent Gas Mixtures, J. Quant. Spectrosc. Radiat. Transfer, vol. 65, pp. 655-672, 2000.

18. A. A. Lacis and V. Oinas, A Description of the Correlated- $k$ Distribution Method for Modeling Nongray Gaseous Absorption, Thermal Emission, and Multiple Scattering in vertically inhomogeneous atomspheres, J. Geophys. Res.-Atmospheres, vol. 96, pp. 9027-9063, 1991.

19. V. Goutière, F. Liu and A. Charette, An Assessment of Real-Gas modelling in 2D Enclosures, J. Quant. Spectrosc. Radiat. Transfer, vol. 64, pp. 299-326, 2000.

20. A. Soufiani and J. Taine, High Temperature Gas Radiative Property Parameters of Statistical Narrow-Band Model for $\mathrm{H}_{2} \mathrm{O}, \mathrm{CO}_{2}$ and $\mathrm{CO}$, and Correlated- $k$ Model for $\mathrm{H}_{2} \mathrm{O}$ and $\mathrm{CO}_{2}$, Int. J. Heat Mass Transfer, vol. 40, pp. 987-991, 1997.

21. W. H. Reed and T. R. Hill, Triangular Mesh Method for the Neutron Transport Equation, Tech. Rep. LA-UR-73-479, Los Alamos Scientific Laboratory Report, Los Alamos, NM, 1973.

22. J. Hesthaven and T. Warburton, Nodal Discontinuous Galerkin Methods: Algorithms, Analysis, and Applications, Springer-Verlag, New York, 2008.

23. X. Cui and B. Q. Li, A Discontinuous Finite-Element Formulation for Multidimensional Radiative Transfer in Absorbing, Emitting, and Scattering Media, Numer. Heat Transfer $B$, vol. 46, pp. 399-428, 2004.

24. C. Aussourd, Styx: A Multidimensional AMR $S_{n}$ Scheme, Nucl. Sci. Eng., vol. 143, pp. 281-290, 2003.

25. C. Fuhrer and R. Rannacher, Error Analysis for the Finite Element Approximation of a Radiative Transfer Model, RAIRO Math. Model. Numer. Anal., vol. 30, pp. 743-762, 1996.

26. J. M. Zhao and L. H. Liu, Discontinuous Spectral Element Method for Solving Radiative Heat Transfer in Multidimensional Semitransparent Media, J. Quant. Spectrosc. Radiat. Transfer, vol. 107, pp. 1-16, 2007. 
27. X. Cui and B. Q. Li, Discontinuous Finite Element Solution of 2-D Radiative Transfer with and without Axisymmetry, J. Quant. Spectrosc. Radiat. Transfer, vol. 96, pp. 383407, 2005.

28. A. T. Patera, A Spectral Element Method for Fluid Dynamics Laminar-Flow in a Channel Expansion, J. Comput. Phys., vol. 54, pp. 468-488, 1984.

29. O. Balima, A. Charette, and D. Marceau, Comparison of Light Transport Models Based on Finite Element and the Discrete Ordinates Methods in View of Optical Tomography Applications, J. Comput. Appl. Math., vol. 234, pp. 2259-2271, 2010.

30. O. Balima, Y. Favennec, J. Boulanger, and A. Charette, Optical Tomography with the Discontinuous Galerkin Formulation of the Radiative Transfer Equation in Frequency Domain, J. Quant. Spectrosc. Radiat. Transfer, vol. 113, pp. 805-814, 2012.

31. N. Bell and M. Garland, Efficient Sparse Matrix-Vector Multiplication on CUDA, NVIDIA Tech. Rep., 2008.

32. E. Elsen, P. LeGresley, and E. Darve, Large Calculation of the Flow Over a Hypersonic Vehicle Using a GPU, J. Comput. Phys., vol. 227, pp. 10148-10161, 2008.

33. A. Corrigan, F. Camelli, R. Lohner, and J. Wallin, Running Unstructured Grid-Based CFD Solvers on Modern Graphics Hardware, Int. J. Numer Meth. Fluids, vol. 66, pp. 221-229, 2011.

34. E. H. Phillips, Y. Zhang, R. L. Davis, and J. D. Owens, Rapid Aerodynamic Performance Prediction on a Cluster of Graphics Processing Units, Proc. 49th AIAA Aerospace Sciences Meeting and Exhibit, vol. 565, 2009.

35. NVIDIA, CUDA Computed Unified Device Architecture, Programming Guide, Version 2.2, 2009.

36. R. Barrett, M. Berry, T. F. Chan, J. Demmel, J. Donato, J. Dongarra, V. Eijkhout, R. Pozo, C. Romine, and H. Van der Vorst, Templates for the Solution of Linear Systems: Building Blocks for Iterative Methods, 22 Ed., SIAM, Philadelphia, PA, 1994.

37. Y. Saad, Iterative Methods for Sparse Linear Systems, SIAM, Philadelphia, PA, 2003.

38. F. H. Lee, K. K. Phoon, K. C. Lim, and S. H. Chan, Performance of Jacobi Preconditioning in Krylov Subspace Solution of Finite Element Equations, Int. J. Numer. Anal. Meth. Geomech., vol. 26, pp. 341-372, 2002.

39. Khronos OpenCL Working Group, The OpenCL Specification, Version 1.0.29.2008, http://khronos.org/registry/cl/specs/opencl-1.0.29.pdf

40. F. Liu, G. J. Smallwood, and O. L. Gulder, Application of the Statistical Narrow-Band Correlated- $k$ Method to Low-Resolution Spectral Intensity and Radiative Heat Transfer Calculations-Effects of the Quadrature Scheme, Int. J. Heat Mass Transfer, vol. 43, pp. 3119-3135, 2000.

41. P. J. Coelho, Fundamentals of a New Method for the Solution of the Radiative Transfer Equation, Int. J. Thermal Sci., vol. 44, pp. 809-821, 2005.

42. T.-K. Kim and H. Lee, Effect of Anisotropic Scattering on Radiative Heat Transfer in Two-Dimensional Rectangular Enclosures, Int. J. Heat Mass Transfer, vol. 31, pp. 1711-1721, 1988.

43. D. N. Trivic, T. J. O'Brien, and C. H. Amon, Modeling the Radiation of Anisotropically Scattering Media by Coupling Mie Theory with Finite Volume Method, Int. J. Heat Mass Transfer, vol. 47, pp. 5765-5780, 2004.

44. D. N. Trivic and C. H. Amon, Modeling the 3-D Radiation of Anisotropically Scattering Media by Two Different Numerical Methods, Int. J. Heat Mass Transfer, vol. 51, pp. 2711-2732, 2008.

45. P. J. Coelho, J. M. Goncalves, M. G. Carvalho, and D. N. Trivic, Modeling of Radiative Heat Transfer in Enclosures with Obstacles, Int. J. Heat Mass Transfer, vol. 41, pp. $745-$ 756, 1998. 
46. G. Kamel, B. M. Naceur, M. Rachid, and S. Rachid, Formulation and Testing of the $\mathrm{FT}_{n}$ Finite Volume Method for Radiation in 3-D Complex Inhomogeneous Participating Media, J. Quant. Spectrosc. Radiat. Transfer, vol. 98, pp. 425-445, 2006.

47. D. Y. Byun, S. W. Baek, and M. Y. Kim, Investigation of Radiative Heat Transfer in Complex Geometries Using Blocked-Off, Multiblock, and Embedded Boundary Treatments, Numer. Heat Transfer A, vol. 43, pp. 807-825, 2003.

48. S. Jendoubi, H. S. Lee, and T.-K. Kim, Discrete Ordinate Solutions for Radiatively Participating Media in a Cylindrical Enclosure, AIAA J. Thermophys. Heat Transfer, vol. 7, pp. 213-219, 1993.

49. Z. Liu, S. V. Ramakrishnan, B. Bihari, C. Olling, and D. Dominik, Aeroheating Prediction Using the Hybrid Flow Solver ICAT, in 20th AIAA Applied Aerodynamics Conference, June 24-26, 2002, Paper no. AIAA 2002-2936.

50. B. Bihari, D. K. Ota, Z. Liu, and S. V. Ramakrishnan, Multiresolution Method on General Unstructured Meshes, AIAA J., vol. 40, pp. 1323-1330, 2002.

51. K. Y. Szema, Z. Liu, S. V. Ramakrishnan, C. Rowell, S. Joshi, and J. Rodrian, Automated Unstructured Grid CFD Tool for Aerodynamic Design Optimization of Airfoils and Wings, in 43rd AIAA Aerospace Sciences Meeting and Exhibit, January 10-15, 2005, Paper No. AIAA 2005-0452.

52. Z. Liu and S. V. Ramakrishnan, Improved Characteristic Velocity Scale Algorithm for Preconditioned Upwind Schemes on Hybrid Grids, in 23rd AIAA Applied Aerodynamics Conference, January 10-15, 2005, Paper No. AIAA 2005-4961.

53. K. Szema, Z. Liu and R. Munipalli, An efficient GUI Design Tool for High-Speed Airbreathing Propulsion Integration, in 28th AIAA Applied Aerodynamics Conference, Chicago, IL, 28 June-1 July, 2010, Paper No. AIAA 2010-4362.

54. P. L. Roe, Approximate Riemann Solvers, Parameter Vectors, and Difference Schemes, J. Comput. Phys., vol. 43, pp. 357-372, 1981.

55. A. Harten, On a Class of High Resolution Total-Variation Stable Finite Difference Schemes, SIAM J. Numer. Anal., vol. 21, pp. 1-23, 1984.

56. S. R. Chakravarthy and S. Osher, A New Class of High Accuracy TVD Schemes for Hyperbolic Conservation Laws, in AIAA paper No. 85-0363, 1985.

57. P. L. Roe, Some Contributions to the Modelling of Discontinuous Flows, Lect. Appl. Math., vol. 22, 1985.

58. F. R. Menter, Eddy Viscousity Transport Equations and Their Relation to the $k$-e Mode, Tech. Rep. 108854, Ames Research Center, Moffett Field, CA, November 1994.

59. S. Palaniswamy, S. R. Chakravarthy, and D. K. Ota, Finite Rate Chemistry for USA-Series Codes: Formulation and Applications, in 27th Aerospace Sciences Meeting, January 19-12, 1989, Paper No. AIAA 89-0200.

60. A. Moore, Efficient memory-based learning for robot control, Ph.D. thesis, University of Cambridge, UK, 1991.

61. F. S. Simmons, Rocket Exhaust Plume Phenomenology, Aerospace Press, EL Segundo, CA, 2000.

62. J. W. Cox, V. Calia, M. Slack, A. Grillo, W. Konopka, W. Ryan, J. Leng, B. Green, B. Upschutte, and K. Holtclaw, Standard Plume Ultraviolet Code (SPURC), Tech. Rep. PL-TR-91-3004, Phillips Lab, Edwards AFB, CA, 1991.

63. A. Berk, G. P. Anderson, P. K. Acharya, L. S. Bernstein, L. Muratov, J. Lee, M. Fox, S. M. Adler-Golden, J. H. Chetwynd, M. L. Hoke, R. B. Lockwood, J. A. Gardner, T. W. Cooley, C. C. Borel, P. E. Lewis, and E. P. Shettle, MODTRAN5: 2006 Update, Proc. SPIE, vol. 6233, $62331 \mathrm{~F}, 2006$.

64. F. Liu and G. J. Smallwood, An Efficient Approach for the Implementation of the SNB Based Correlated-k Method and Its Evaluation, J. Quant. Spectrosc. Radiat. Transfer, vol. 84, pp. 465-475, 2004. 